\title{
Transient Analysis of Sharp Thermal Gradients Using Coarse Finite Element Meshes
}

\author{
P. O'Hara ${ }^{\dagger}$, C.A. Duarte ${ }^{\dagger}$ and T. Eason \\ ${ }^{\dagger}$ Department of Civil and Environmental Engr., University of Illinois at Urbana-Champaign, \\ Newmark Laboratory, 205 North Mathews Avenue, Urbana, Illinois 61801, USA \\ e-mails: \{pohara,caduarte\}@illinois.edu \\ ¥Air Force Research Laboratory \\ Air Vehicles Directorate, WPAFB, Ohio, USA, \\ e-mail: Thomas.Eason@wpafb.af.mil
}

July 26, 2010

\begin{abstract}
This paper investigates the application of the Generalized Finite Element Method with global-local enrichments $\left(G F E M^{\mathrm{gl}}\right)$ to problems of transient heat transfer involving localized features. The $G F E M^{\mathrm{gl}}$ is utilized in order to numerically construct general, specially-tailored shape functions yielding high levels of accuracy on coarse FEM meshes. The use of time-dependent shape functions requires that the system of equations be discretized temporally first, and then spatially in order to properly account for the time-dependency. The standard $\alpha$-method is used for the time integration scheme. The transient three-dimensional FFEM $^{\mathrm{gl}}$ is then applied to a laser heating example in order to demonstrate its ability to resolve localized, transient features on a fixed, coarse mesh. Convergence analysis of the proposed method and as well as applications to heterogeneous materials, and moving heat sources are also provided.
\end{abstract}

\section{Introduction}

Many applications areas in engineering practice involve the analysis of structural behavior with multiple scales of interest. The analysis of structures subjected to intense, time-dependent, localized thermal loadings is the subject of interest in this work. The motivation for this particular investigation is the analysis of localized heat sources on the skin of hypersonic flight vehicles. Possible interactions of shock waves can cause severe, localized thermal loadings, exhibiting sharp gradients. The characterization of the resulting thermal loadings and pressure distributions, as well as the effect of these loadings on the aeroelastic behavior of the vehicle itself has been an active topic of research in recent years [11, 21, 22, 30, 39, 40, 43]. The most severe potential shock wave interference pattern is the Edney Type IV shock wave interaction, which is a bow shock/cowl shock interaction which may occur on the leading edge of a wing.

While hypersonic flight vehicles is the motivating case for this work, the methodology proposed here may be applied to any of a number of practical situations involving intense, and highly localized heat sources. Numerical modeling of laser heating $[45,46]$ of metal plates and the resulting thermo-mechanical effects of the intense localized heat source is another potential are of application. Computational weld mechanics $[26,44]$ is yet another potential application area of interest, in which only a small portion of a much larger 
structural element is subjected to a very localized heat source. In the afore-mentioned application areas the use of highly refined meshes is required to resolve the localized temperature fields, resulting in active research into methods which are more computationally efficient (particularly for fully 3-D simulations), and do not require the immense computational resources to run simulations requiring excessively high mesh density.

We propose to use the Generalized Finite Element Method with global-local enrichment functions (GFEM ${ }^{\mathrm{gl}}$ ) [14] to circumvent the need for highly refined meshes, and resolve the sharp localized gradients on fixed, coarse meshes, thus making the 3-D transient analysis of such scenarios more computationally feasible. The work presented in this paper is an extension of the methodology developed in [32], extended for the analysis of time-dependent problems.

The proposed method is not unique in the use of time-dependent shape functions, as numerous other methods also allow for the evolution of shape functions in time. The shape functions used in [29] evolve to follow a thermal spike as it propagates throughout the domain. van der Meer et al. [41] use time-dependent shape functions for geothermal applications involving sharp gradients. Two methods, one based on a simplified analytic solution, and the other, an iterative optimization method to properly parameterize a Gaussian shape function are presented in order to construct specially-tailored shape functions to solve large-scale problems that involve very localized behavior. In this way, their analysis is similar to those presented in this research, but a major difference is found in the construction of the special enrichment functions. In this work we numerically build appropriate enrichment functions based on the solution of local boundary value problems; a procedure which may be used when no a-priori knowledge about the solution is available.

Time-dependent shape functions are also utilized by Waisman and Belytchsko [42] to efficiently solve boundary layer problems by adapting the shape functions to reflect the underlying physics of the problem that is to be solved. Chessa and Belytschko [8] solve the problem of axisymmetric two-phase flow in which a time-dependent level-set function is used in order to track the moving interface between the fluids. Time-dependent level-sets are again used by Chessa and Belytschko [9] within a space-time finite element context in order to solve the linear wave equation as well as Burger's equation. Fries and Zilian [23] offer a detailed formulation for the use of time-dependent enrichment functions in a semi-discrete finite element context. They solve Burger's equation along with the advection-diffusion equation using both space-time finite elements as well as the semi-discrete finite element method utilizing time-dependent shape functions. Fries and Zilian offer a detailed convergence analysis with respect to spatial as well as temporal errors.

In the next section, we discuss the formulation of the governing equations, i.e. the heat equation. A brief presentation of generalized finite element (GFEM) approximations [3, 4, 15, 31, 38] is provided in Section 3. Section 4 presents the discrete system of equations to be solved as well as the time integration algorithm used. Section 5 provides a detailed account of the model problem to be investigated in this paper. Output obtained from standard FEM [34, 47] is presented along with data obtained from GFEM analyses of the same problem. Motivated by the potential to solve the problem using specially-designed, but more general shape functions, we present the formulation of the Generalized Finite Element Method with global-local enrichments $\left(G F E M^{\mathrm{gl}}\right)$ for time-dependent heat transfer problems in Section 6. Section 7 provides detailed analyzes of the model problem and two other numerical examples, using the $G F E M^{\mathrm{gl}}$ to study the method's ability to effectively control the error in the solution. The final section then provides the main conclusions and future directions for the current investigation. 


\section{Problem Formulation}

Consider a domain $\Omega \subset \boldsymbol{R}^{3}$ with boundary $\partial \Omega$ decomposed as $\partial \Omega=\Gamma_{c} \cup \Gamma_{f}$ with $\Gamma_{c} \cap \Gamma_{f}=\emptyset$. The strong form of the governing equation is given by the 3 -D heat equation

$$
\rho c \frac{\partial u}{\partial t}=\nabla(\boldsymbol{\kappa} \nabla u)+Q(\boldsymbol{x}, t) \quad \text { in } \quad \Omega
$$

where $u(\boldsymbol{x}, t)$ is the temperature field, $\rho c$ is the volumetric heat capacity and $Q(\boldsymbol{x}, t)$ is the internal heat source. In the general case, $\boldsymbol{K}$ is the thermal conductivity tensor, but in this paper, only isotropic materials are considered, therefore the thermal conductivity is merely a scalar, $\kappa=\kappa(\boldsymbol{x})$, and the material need not be homogeneous.

We consider convective and Neumann boundary conditions prescribed on $\Gamma_{c}$ and $\Gamma_{f}$, respectively. The boundary $\partial \Omega=\Gamma_{c} \cup \Gamma_{f}$ and $\Gamma_{c} \cap \Gamma_{f}=\emptyset$. At any time $t$, the normal flux is prescribed as

$$
\begin{gathered}
-\kappa \frac{\partial u}{\partial n}=\eta(\bar{u}-u) \quad \text { on } \quad \Gamma_{c} \\
-\kappa \frac{\partial u}{\partial n}=\bar{f} \quad \text { on } \quad \Gamma_{f}
\end{gathered}
$$

where $\bar{u}$ and $\eta$ are the prescribed fluid temperature and convective coefficient, respectively.

Dirichlet boundary conditions can be treated as a limiting case of convective boundary conditions by selecting a large value for the convective coefficient $\eta$. This leads to the well known penalty method [2].

The initial conditions must also be satisfied

$$
u(\boldsymbol{x}, 0)=u^{0}(\boldsymbol{x}) \quad \text { at } \quad t^{0}
$$

where $u^{0}(\boldsymbol{x})$ is the prescribed temperature field at time $t=t^{0}$.

\section{Generalized FEM Approximations}

Historically, polynomials have been used to approximate functions and it has been shown that they have good approximation properties. Methods based on polynomials, e.g., the Finite Element Method (FEM), have been successfully used to approximate solutions of various types of PDEs. Nonetheless, classical methods like the FEM require extremely refined meshes to approximate the solution of the class of problems considered in this paper. In contrast, the Generalized Finite Element Method (GFEM) is a Galerkin method that allows the use of non-polynomial approximating functions. These approximating functions can be carefully chosen to mimic the properties of the unknown function to be approximated. The GFEM [3, 15, $31,38]$ is an instance of the partition of unity method which has its origins in the works of Babuška et al. $[3,4,28]$ (under the names "special finite element methods", "generalized finite element method" and "finite element partition of unity method") and Duarte and Oden [12, 18-20, 31] (under the names " $h p$ clouds" and "cloud-based $h p$ finite element method"). Several meshfree methods can also be formulated as special cases of the partition of unity method.

The construction of the approximation space (i.e., the trial space) to be used in the GFEM consists of three components - (a) patches or clouds, (b) a partition of unity, and (c) the local approximation spaces. We describe these components as follows:

(a) Patches or Clouds: For a parameter $h>0$, let $\left\{\omega_{\alpha}\right\}_{\alpha=1}^{N(h)}$ be sub-domains of the underlying domain 
$\Omega \subset \mathbb{R}^{d}$ of interest. We assume that for each value of $h$,

$$
\Omega=\cup_{\alpha=1}^{N} \omega_{\alpha},
$$

and that any $\boldsymbol{x} \in \Omega$ belongs to at most $M$ of the sets $\omega_{\alpha}$, where $M$ is independent of $h$. The sub-domains $\omega_{\alpha}$ are called patches or clouds. In the generalized finite element method, a $\omega_{\alpha}$ is given by the union of the finite elements sharing node $\alpha$ of the finite element mesh covering $\Omega$. Clearly, $\left\{\omega_{\alpha}\right\}_{\alpha=1}^{N}$ is an open cover of $\Omega$, and each patch $\omega_{\alpha}$ intersects with at most $M$ of other patches $\omega_{\alpha}$.

(b) Partition of Unity: Let $\left\{\varphi_{\alpha}\right\}_{j=1}^{N}$ be piecewise $C^{1}$ functions defined on $\Omega$ satisfying

$$
\sum_{\alpha=1}^{N} \varphi_{\alpha}(\boldsymbol{x})=1, \quad \forall \boldsymbol{x} \in \Omega
$$

It is clear that $\left\{\varphi_{\alpha}\right\}_{\alpha=1}^{N}$ is a partition of unity with respect to the open cover $\left\{\omega_{\alpha}\right\}_{\alpha=1}^{N}$.

(c) Local Approximation Spaces: To each patch $\omega_{\alpha}$, we associate an $m_{\alpha}$-dimensional space $\chi_{\alpha}$ of functions defined on $\omega_{\alpha}$, namely,

$$
\chi_{\alpha}=\operatorname{span}\left\{L_{\alpha i}, 1 \leq i \leq m_{\alpha}, L_{\alpha i} \in H^{1}\left(\omega_{\alpha}\right)\right\} .
$$

$\chi_{\alpha} \mathrm{s}$ are called local approximation spaces. We assume that each $\chi_{\alpha}$ contains constant functions. The functions in $\chi_{\alpha}$ are also known as enrichment functions.

The trial space for the GFEM is given by

$$
S^{G F E M}(\Omega) \equiv \sum_{\alpha=1}^{N} \varphi_{\alpha} \chi_{\alpha}=\operatorname{span}\left\{\phi_{\alpha i}:=\varphi_{\alpha} L_{\alpha i}, 1 \leq i \leq m_{\alpha}, 1 \leq \alpha \leq N\right\}
$$

The function

$$
\phi_{\alpha i}(\boldsymbol{x})=\varphi_{\alpha}(\boldsymbol{x}) L_{\alpha i}(\boldsymbol{x}) \quad(\text { no summation on } \alpha),
$$

where $\alpha$ is a node in the finite element mesh, is called a GFEM shape function. Figure 1 illustrates the construction of GFEM shape functions in a two-dimensional domain.

Enrichment functions We first note that the role of the partition of unity is to "paste together" the functions in $\chi_{\alpha}$, which are defined locally only on $\omega_{\alpha}$, to obtain a global function in $H^{1}(\Omega)$. The enrichment functions in $\chi_{\alpha}$ are chosen carefully to mimic the properties of the function to be approximated, locally in $\omega_{\alpha}$. This is often done by using the available information on the approximated function. Choosing suitable local approximation spaces for a particular problem is central to the approximation property of GFEM. This is particularly clear from the main approximation result of $\operatorname{GFEM}[3,4,28]$, which could also be stated as (see [6]):

Theorem: Let $u \in H^{1}(\Omega)$. Then there is $u_{h p} \in S^{G F E M}$ such that

$$
\left\|u-u_{h p}\right\|_{H^{1}(\Omega)}^{2} \leq C \sum_{\alpha=1}^{N} \inf _{u_{\alpha} \in \chi_{\alpha}}\left\|u-u_{\alpha}\right\|_{H^{1}\left(\omega_{\alpha}\right)}^{2}
$$

It is clear from the above result that the quality of the global approximation $u_{h p}$ of $u$ is governed by the quality of the local approximation of $u$ by the functions in $\chi_{\alpha}$.

The GFEM has been successfully applied to the simulation of boundary layers [13], dynamic propagating fractures [16], singularities [15], acoustic problems with high wave number [5, 27], polycrystalline mi- 


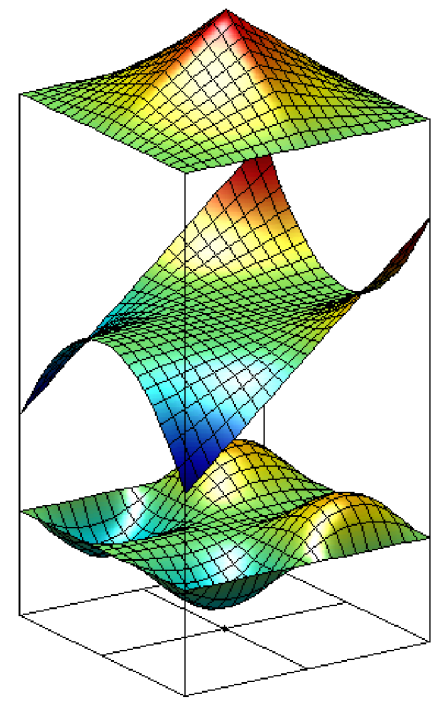

(a)

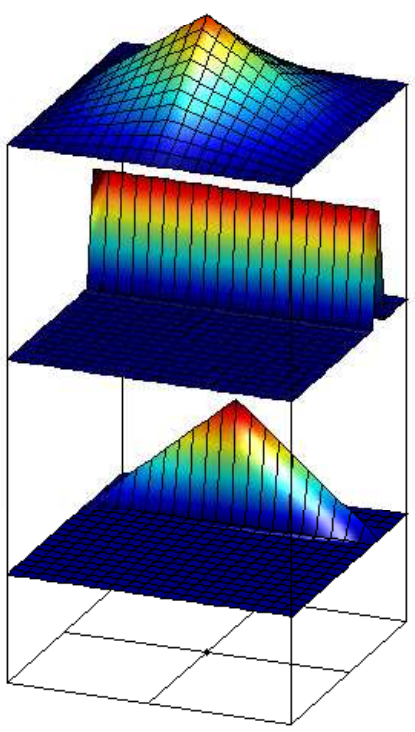

(b)

Figure 1: Construction of a generalized FEM shape function using a polynomial (a) and a non-polynomial enrichment (b). Here, $\varphi_{\alpha}$ are the functions at the top, the enrichment functions, $L_{\alpha i}$, are the functions in the middle, and the generalized $F E$ shape functions, $\phi_{\alpha i}$, are the resulting bottom functions.

crostructures [36], porous materials [37], etc. All these applications rely on enrichment functions in $\chi_{\alpha}$ derived analytically using a-priori knowledge about the functions to be approximated. These so-called custom enrichment functions are able to provide more accurate and robust simulations than the polynomial functions traditionally used in the standard FEM while relaxing some meshing requirements of the FEM. However, for many classes of problems-like those considered in this paper-enrichment functions with good approximation properties are, in general, not amenable to analytical derivation. In this paper, we propose to remove the limitations of existing generalized finite element methods for the solution of time-dependent problems exhibiting highly localized sharp, transient thermal gradients. Details are provided in Section 6.

\section{Time Integration and Discrete Equations}

In this section we discretize (1) in a finite element context. In the first formulation we discretize the equations first in space, and then in time. With this formulation strategy, the algorithm is appropriate for analyses which do not include time-dependencies in the shape functions. For the case with time-dependent shapefunctions it is important to discretize the equations first in time, and then in space, as is discussed in [8, 23, 41]. The formulation for time-dependent shape functions is subsequently presented in 4.2.

\subsection{Formulation 1: Discretizing Heat Equation Spatially, then Temporally}

A standard formulation for time-integration of first order, parabolic equations can be found in many finite element method books, one such instance is [35]. The formulation in [35], given by Reddy starts with a system of spatially discretized equations as in (6), and utilizes the finite difference assumption in (7).

$$
\mathbf{M} \dot{u}^{n+1}+\mathbf{K} \boldsymbol{u}^{n+1}=\boldsymbol{f}^{n+1}
$$




$$
\boldsymbol{u}^{n+1}=\boldsymbol{u}^{n}+\Delta t\left[\alpha \dot{\boldsymbol{u}}^{n+1}+(1-\alpha) \dot{\boldsymbol{u}}^{n}\right]
$$

Equation (7) is used to eliminate $\dot{\boldsymbol{u}}^{n+1}$ from (6), yielding the discrete system of equations used for timeintegration:

$$
[\mathbf{M}+\alpha \Delta t \mathbf{K}] \boldsymbol{u}^{n+1}=[\mathbf{M}-(1-\alpha) \Delta t \mathbf{K}] \boldsymbol{u}^{n}+\Delta t\left[\alpha \boldsymbol{f}^{n+1}+(1-\alpha) \boldsymbol{f}^{n}\right]
$$

where terms associated with convective boundary conditions are omitted. The potential draw-back of this particular formulation is that it starts from a spatially-discretized system, in which the quantities have previously been defined as

$$
\begin{gathered}
\mathbf{M}=\int_{\Omega} \boldsymbol{\phi} \rho c(\boldsymbol{\phi})^{T} d \Omega \\
\mathbf{K}=\int_{\Omega} \nabla \boldsymbol{\phi} \kappa(\nabla \boldsymbol{\phi})^{T} d \Omega \\
\boldsymbol{f}^{n}=\boldsymbol{f}_{Q}^{n}+\boldsymbol{f}_{N}^{n}
\end{gathered}
$$

where $\boldsymbol{\phi}$ is the vector of finite element shape functions, $\Omega$ is the domain of interest and $\boldsymbol{u}^{n+1}$ is the solution vector at $t=t^{n+1}=(n+1) \Delta t$. In (11) the quantities are defined as

$$
\begin{aligned}
\boldsymbol{f}_{Q}^{n} & =\int_{\Omega} Q^{n} \boldsymbol{\phi} d \Omega \\
\boldsymbol{f}_{N}^{n} & =\int_{\Gamma_{f}} \bar{f}^{n} \boldsymbol{\phi} d \Gamma
\end{aligned}
$$

where $Q^{n}=Q\left(\boldsymbol{x}, t^{n}\right)$ and $\bar{f}^{n}=\bar{f}\left(\boldsymbol{x}, t^{n}\right)$.

The previous formulation is the widely used $\alpha$-method, suitable for transient heat transfer simulations, but the formulation is in no way modified to incorporate the use of time-dependent shape functions.

\subsection{Formulation 2: Discretizing Heat Equation Temporally, then Spatially}

In this section we discretize the heat equation first in time, then in space [8, 23, 41]. With this formulation, the algorithm is appropriate for the use of time-dependent shape functions. We start our formulation with the strong form of the governing equation:

$$
\rho c \frac{\partial u}{\partial t}=\nabla \cdot \kappa \nabla u+Q
$$

We then multiply the equation by a weighting function, $w$, and integrate over the domain, $\Omega$.

$$
\int_{\Omega} w \rho c \frac{\partial u}{\partial t} d \Omega=\int_{\Omega}(w \nabla \cdot \kappa \nabla u+w Q) d \Omega
$$

We perform integration by parts on the first term of the right-hand-side, and move the domain integral to the left-hand-side of the equation. The boundary term is left on the right-hand-side with the applied source term.

$$
\int_{\Omega}\left(w \rho c \frac{\partial u}{\partial t}+\nabla w \cdot \kappa \nabla u\right) d \Omega=\int_{\partial \Omega} w \kappa \frac{\partial u}{\partial n} d \Gamma+\int_{\Omega} w Q d \Omega
$$

We now discretize the equation above in time. To this end we will use the following finite difference 
approximations, yielding the generalized trapezoidal rule, or $\alpha$-method, used for our time-marching scheme.

$$
\begin{gathered}
\frac{\partial u}{\partial t}=\frac{u^{n+1}-u^{n}}{\Delta t} \\
u^{n+\alpha}=(1-\alpha) u^{n}+\alpha u^{n+1}
\end{gathered}
$$

Plugging (15) and (16) into (14) yields the temporally discretized equation

$$
\begin{aligned}
& \int_{\Omega}\left(w \rho c \frac{u^{n+1}-u^{n}}{\Delta t}+\nabla w \cdot \kappa\left[\alpha \nabla u^{n+1}+(1-\alpha) \nabla u^{n}\right]\right) d \Omega \\
& =\int_{\Omega} w\left[\alpha Q^{n+1}+(1-\alpha) Q^{n}\right] d \Omega+\int_{\partial \Omega} w \kappa\left[\alpha \frac{\partial u^{n+1}}{\partial n}+(1-\alpha) \frac{\partial u^{n}}{\partial n}\right] d \Gamma
\end{aligned}
$$

We will first consider, in detail, the boundary terms on $\partial \Omega=\Gamma_{c} \bigcup \Gamma_{f}$ as follows:

$$
\begin{aligned}
& \int_{\partial \Omega} w \kappa\left[\alpha \frac{\partial u^{n+1}}{\partial n}+(1-\alpha) \frac{\partial u^{n}}{\partial n}\right] d \Gamma=\alpha \int_{\Gamma_{f}} w \bar{f}^{n+1} d \Gamma+(1-\alpha) \int_{\Gamma_{f}} w \bar{f}^{n} d \Gamma+\alpha \int_{\Gamma_{c}} w \eta \bar{u}^{n+1} d \Gamma \\
& -\alpha \int_{\Gamma_{c}} w \eta u^{n+1} d \Gamma+(1-\alpha) \int_{\Gamma_{c}} w \eta \bar{u}^{n} d \Gamma-(1-\alpha) \int_{\Gamma_{c}} w \eta u^{n} d \Gamma
\end{aligned}
$$

We now rearrange (17) with the proper boundary terms (18) such that terms involving $u^{n+1}$ are moved to the left-hand-side, and all known terms (those not dependent upon $u^{n+1}$ ) are moved to the right-hand-side.

$$
\begin{aligned}
& \frac{1}{\Delta t} \int_{\Omega} w \rho c u^{n+1} d \Omega+\alpha \int_{\Omega} \nabla w \cdot \kappa \nabla u^{n+1} d \Omega+\alpha \int_{\Gamma_{c}} w \eta u^{n+1} d \Gamma \\
& =\frac{1}{\Delta t} \int_{\Omega} \rho c w u^{n} d \Omega-(1-\alpha) \int_{\Omega} \nabla w \cdot \kappa \nabla u^{n} d \Omega+\alpha \int_{\Omega} w Q^{n+1} d \Omega+(1-\alpha) \int_{\Omega} w Q^{n} d \Omega \\
& +\alpha \int_{\Gamma_{f}} w \bar{f}^{n+1} d \Gamma+(1-\alpha) \int_{\Gamma_{f}} w \bar{f}^{n} d \Gamma+\alpha \int_{\Gamma_{c}} w \eta \bar{u}^{n+1} d \Gamma+(1-\alpha) \int_{\Gamma_{c}} w \eta \bar{u}^{n} d \Gamma \\
& -(1-\alpha) \int_{\Gamma_{c}} w \eta u^{n} d \Gamma
\end{aligned}
$$

At this point, we have our system of equations fully discretized in time. For the spatial discretization, we use generalized finite element shape functions which may have time-dependencies. At any given time, $t^{n}$, we define $u^{n}\left(\boldsymbol{x}, t^{n}\right)=\boldsymbol{\phi}^{n}\left(\boldsymbol{x}, t^{n}\right) \cdot \boldsymbol{u}^{n}\left(t^{n}\right)$, where $\boldsymbol{u}^{n}\left(t^{n}\right)$ is the vector of degrees of freedom, and $\boldsymbol{\phi}^{n}\left(\boldsymbol{x}, t^{n}\right)$ is the vector of finite element shape functions at $t^{n}$. Due to the potential time-dependent nature of the shape functions, it is very important to properly select the discretization for the weight function, $w[23,41]$. In our implementation, we need $w$ to be consistent across each term of (19). To this end, we discretize the weighting function, $w$ using finite element shape functions at time $t^{n+1}$. In the afore mentioned equations, $w=w^{n+1}\left(\boldsymbol{x}, t^{n+1}\right)=\boldsymbol{\phi}^{n+1}\left(\boldsymbol{x}, t^{n+1}\right) \cdot \boldsymbol{w}^{n+1}\left(t^{n+1}\right)$. We will now discretize (19) on a term-by-term basis using the previous discretizations.

$$
\begin{gathered}
\int_{\Omega} \rho c w^{n+1} u^{n+1} d \Omega=\left(\boldsymbol{w}^{n+1}\right)^{T} \int_{\Omega} \boldsymbol{\phi}^{n+1} \rho c\left(\boldsymbol{\phi}^{n+1}\right)^{T} d \Omega \boldsymbol{u}^{n+1}=\left(\boldsymbol{w}^{n+1}\right)^{T} \mathbf{M}^{n+1} \boldsymbol{u}^{n+1} \\
\int_{\Omega} \rho c w^{n+1} u^{n} d \Omega=\left(\boldsymbol{w}^{n+1}\right)^{T} \int_{\Omega} \boldsymbol{\phi}^{n+1} \rho c\left(\boldsymbol{\phi}^{n}\right)^{T} d \Omega \boldsymbol{u}^{n}=\left(\boldsymbol{w}^{n+1}\right)^{T} \mathbf{M}^{n+1, n} \boldsymbol{u}^{n}
\end{gathered}
$$




$$
\begin{aligned}
& \int_{\Omega} \nabla w^{n+1} \cdot \kappa \nabla u^{n+1} d \Omega=\left(\boldsymbol{w}^{n+1}\right)^{T} \int_{\Omega} \nabla \boldsymbol{\phi}^{n+1} \kappa\left(\nabla \boldsymbol{\phi}^{n+1}\right)^{T} d \Omega \boldsymbol{u}^{n+1}=\left(\boldsymbol{w}^{n+1}\right)^{T} \mathbf{K}^{n+1} \boldsymbol{u}^{n+1} \\
& \int_{\Omega} \nabla w^{n+1} \cdot \kappa \nabla u^{n} d \Omega=\left(\boldsymbol{w}^{n+1}\right)^{T} \int_{\Omega} \nabla \boldsymbol{\phi}^{n+1} \kappa\left(\nabla \boldsymbol{\phi}^{n}\right)^{T} d \Omega \boldsymbol{u}^{n}=\left(\boldsymbol{w}^{n+1}\right)^{T} \mathbf{K}^{n+1, n} \boldsymbol{u}^{n} \\
& \int_{\Omega} w^{n+1} Q^{n+1} d \Omega=\left(\boldsymbol{w}^{n+1}\right)^{T} \int_{\Omega} \boldsymbol{\phi}^{n+1} Q^{n+1} d \Omega=\left(\boldsymbol{w}^{n+1}\right)^{T} \boldsymbol{f}_{Q}^{n+1} \\
& \int_{\Omega} w^{n+1} Q^{n} d \Omega=\left(\boldsymbol{w}^{n+1}\right)^{T} \int_{\Omega} \boldsymbol{\phi}^{n+1} Q^{n} d \Omega=\left(\boldsymbol{w}^{n+1}\right)^{T} \boldsymbol{f}_{Q}^{n+1, n} \\
& \int_{\Gamma_{f}} w^{n+1} \bar{f}^{n+1} d \Gamma=\left(\boldsymbol{w}^{n+1}\right)^{T} \int_{\Gamma_{f}} \boldsymbol{\phi}^{n+1} \bar{f}^{n+1} d \Gamma=\left(\boldsymbol{w}^{n+1}\right)^{T} \boldsymbol{f}_{N}^{n+1} \\
& \int_{\Gamma_{f}} w^{n+1} \bar{f}^{n} d \Gamma=\left(\boldsymbol{w}^{n+1}\right)^{T} \int_{\Gamma_{f}} \boldsymbol{\phi}^{n+1} \bar{f}^{n} d \Gamma=\left(\boldsymbol{w}^{n+1}\right)^{T} \boldsymbol{f}_{N}^{n+1, n} \\
& \int_{\Gamma_{c}} \eta w^{n+1} u^{n+1} d \Gamma=\left(\boldsymbol{w}^{n+1}\right)^{T} \int_{\Gamma_{c}} \boldsymbol{\phi}^{n+1} \eta\left(\boldsymbol{\phi}^{n+1}\right)^{T} d \Gamma \boldsymbol{u}^{n+1}=\left(\boldsymbol{w}^{n+1}\right)^{T} \mathbf{M}_{c}^{n+1} \boldsymbol{u}^{n+1} \\
& \int_{\Gamma_{c}} \eta w^{n+1} u^{n} d \Gamma=\left(\boldsymbol{w}^{n+1}\right)^{T} \int_{\Gamma_{c}} \boldsymbol{\phi}^{n+1} \eta\left(\boldsymbol{\phi}^{n}\right)^{T} d \Gamma \boldsymbol{u}^{n}=\left(\boldsymbol{w}^{n+1}\right)^{T} \mathbf{M}_{c}^{n+1, n} \boldsymbol{u}^{n} \\
& \int_{\Gamma_{c}} \eta w^{n+1} \bar{u}^{n+1} d \Gamma=\left(\boldsymbol{w}^{n+1}\right)^{T} \int_{\Gamma_{c}} \boldsymbol{\phi}^{n+1} \eta \bar{u}^{n+1} d \Gamma=\left(\boldsymbol{w}^{n+1}\right)^{T} \boldsymbol{f}_{c}^{n+1} \\
& \int_{\Gamma_{c}} \eta w^{n+1} \bar{u}^{n} d \Gamma=\left(\boldsymbol{w}^{n+1}\right)^{T} \int_{\Gamma_{c}} \boldsymbol{\phi}^{n+1} \eta \bar{u}^{n} d \Gamma=\left(\boldsymbol{w}^{n+1}\right)^{T} \boldsymbol{f}_{c}^{n+1, n}
\end{aligned}
$$

Since equation (19) must hold for any admissible weight function $w$, it must hold also for any $\boldsymbol{w}^{n+1}$. As such, we can pose the fully discretized system of equations as

$$
\begin{aligned}
& {\left[\frac{1}{\Delta t} \mathbf{M}^{n+1}+\alpha \mathbf{K}^{n+1}+\alpha \mathbf{M}_{c}^{n+1}\right] \boldsymbol{u}^{n+1}=\left[\frac{1}{\Delta t} \mathbf{M}^{n+1, n}-(1-\alpha) \mathbf{K}^{n+1, n}-(1-\alpha) \mathbf{M}_{c}^{n+1, n}\right] \boldsymbol{u}^{n}} \\
& +\alpha \boldsymbol{f}_{Q}^{n+1}+(1-\alpha) \boldsymbol{f}_{Q}^{n+1, n}+\alpha \boldsymbol{f}_{N}^{n+1}+(1-\alpha) \boldsymbol{f}_{N}^{n+1, n}+\alpha \boldsymbol{f}_{c}^{n+1}+(1-\alpha) \boldsymbol{f}_{c}^{n+1, n}
\end{aligned}
$$

More concisely, we can re-write the above equation as:

$$
\left[\frac{1}{\Delta t} \mathbf{M}^{n+1}+\alpha \hat{\mathbf{K}}^{n+1}\right] \boldsymbol{u}^{n+1}=\left[\frac{1}{\Delta t} \mathbf{M}^{n+1, n}-(1-\alpha) \hat{\mathbf{K}}^{n+1, n}\right] \boldsymbol{u}^{n}+\alpha \hat{\boldsymbol{f}}^{n+1}+(1-\alpha) \hat{\boldsymbol{f}}^{n+1, n}
$$

where

$$
\begin{gathered}
\hat{\mathbf{K}}^{n+1}=\mathbf{K}^{n+1}+\mathbf{M}_{c}^{n+1} \\
\hat{\mathbf{K}}^{n+1, n}=\mathbf{K}^{n+1, n}+\mathbf{M}_{c}^{n+1, n} \\
\hat{\boldsymbol{f}}^{n+1}=\boldsymbol{f}_{Q}^{n+1}+\boldsymbol{f}_{N}^{n+1}+\boldsymbol{f}_{c}^{n+1} \\
\hat{\boldsymbol{f}}^{n+1, n}=\boldsymbol{f}_{Q}^{n+1, n}+\boldsymbol{f}_{N}^{n+1, n}+\boldsymbol{f}_{c}^{n+1, n}
\end{gathered}
$$


In all of the above equations, $\boldsymbol{u}^{n}$ are known values obtained from the solution at $t^{n}$. It is noted that if the shape functions are not time-dependent, $\mathbf{M}^{n+1}=\mathbf{M}^{n+1, n}=\mathbf{M}, \mathbf{K}^{n+1}=\mathbf{K}^{n+1, n}=\mathbf{K}, \boldsymbol{f}_{Q}^{n+1, n}=\boldsymbol{f}_{Q}^{n}, \boldsymbol{f}_{N}^{n+1, n}=\boldsymbol{f}_{N}^{n}$ and $\boldsymbol{f}_{c}^{n+1, n}=\boldsymbol{f}_{c}^{n}$. It is further noted that if the convective boundary terms are omitted, (33) is equivalent to (8).

For the analyzes presented in the subsequent sections, the value of $\alpha$ is taken as $\alpha=1$, yielding the unconditionally stable, Backward Euler algorithm. As such, only the non-symmetric capacity matrix, $\mathbf{M}^{n+1, n}$ is required, and it need not be assembled directly. The vector term $\mathbf{M}^{n+1, n} \boldsymbol{u}^{n}$ can be computed as

$$
\mathbf{M}^{n+1, n} \boldsymbol{u}^{n}=\int_{\Omega} \boldsymbol{\phi}^{n+1} \rho c\left(\boldsymbol{\phi}^{n}\right)^{T} \boldsymbol{u}^{n} d \Omega=\int_{\Omega} \rho c \boldsymbol{\phi}^{n+1} u^{n} d \Omega
$$

where $u^{n}=\left(\boldsymbol{\phi}^{n}\right)^{T} \boldsymbol{u}^{n}$, is the GFEM solution from time step $t^{n}$.

\section{Model Problem}

The problem selected for verification of the GFEM involves a sharp spatial gradient in the temperature field (39), as well as in the resulting source term (40). There is also a temporal gradient, but it is smooth in nature. This particular problem is taken from [29], with the modification that we are assuming a stationary thermal spike.

$$
\begin{gathered}
u(x, t)=\left(\exp ^{-\gamma\left(x-x_{0}\right)^{2}}+\sin \left(\frac{\pi x}{L}\right)\right) * \exp ^{(-t)} \quad 0<x<L \\
Q(x, t)=\rho c \frac{d u}{d t}(x, t)-\kappa \frac{d^{2} u}{d x^{2}}(x, t),
\end{gathered}
$$

The initial and boundary conditions are given in (41) and (42), respectively.

$$
\begin{gathered}
u(x, 0)=\exp ^{-\gamma\left(x-x_{0}\right)^{2}}+\sin \left(\frac{\pi x}{L}\right), \\
u(0, t)=u(L, t)=0,
\end{gathered}
$$

In the above equations, $x_{0}=125 \mathrm{~mm}, L=500 \mathrm{~mm}$ and $\gamma$ is a parameter controlling the roughness of the solution. Unless otherwise indicated, the value of $\gamma$ is taken as 1.0. The material properties are taken as thermal conductivity, $\kappa=1$ and volumetric heat capacity, $\rho c=\left(\frac{\pi}{L}\right)^{2}$. The one-dimensional solution (39) is plotted in Figure 2. The parameter $x_{0}$ defines the location of a thermal spike. From the temporal standpoint, the solution undergoes a smooth, exponential decay in time.

Below, the heat equation with $Q$ given by (40), initial and boundary conditions given in (41) and (42), respectively, is solved using 1-, 2- and 3-D GFEM discretizations.

The exact internal energy, $U(t)$, in the solution domain $\Omega$ is given by

$$
U(t)=\int_{\Omega}(\kappa \nabla u) \cdot(\nabla u) d \Omega
$$

while the internal energy of the GFEM solution at time $t^{n}$ is given by

$$
U_{\mathrm{hp}}\left(t^{n}\right)=\int_{\Omega}\left(\kappa \nabla u^{n}\right) \cdot\left(\nabla u^{n}\right) d \Omega
$$




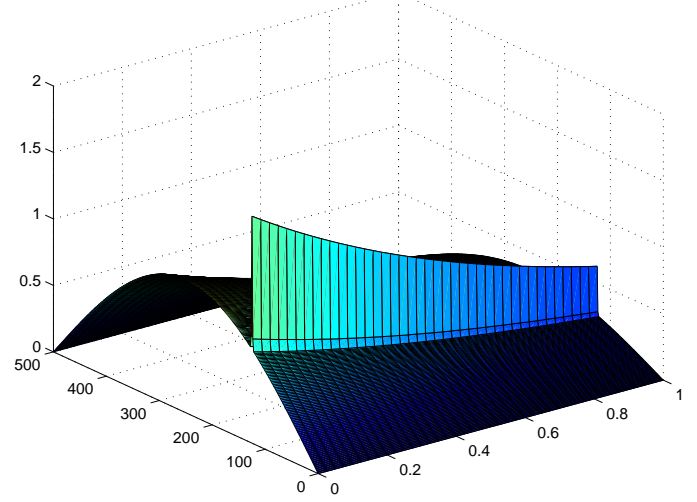

(a) Reference solution in space and time.

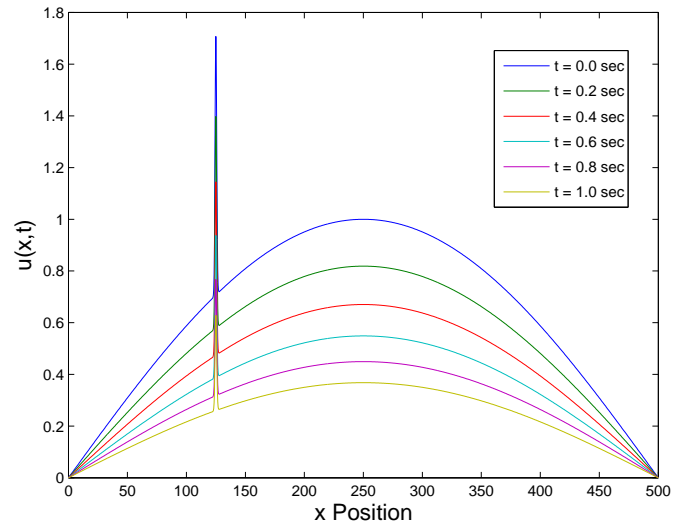

(b) Time slices of reference solution at increasing time, $t$.

Figure 2: Temperature field described in (39).

The discrete $L_{2}$ norm of $U(t), t \in\left[0, t_{\text {final }}\right]$, is defined as

$$
\|U(t)\|_{2}=\left\{\sum_{n}\left(U\left(t^{n}\right)\right)^{2}\right\}^{1 / 2}
$$

where the summation is performed over each time step $t^{n}, \in\left[0, t_{\text {final }}\right]$.

The relative error of $U_{\mathrm{hp}}(t)$ in the discrete $L_{2}$ norm is given by

$$
L_{2}^{\text {Error }}(U(t))=\frac{\left\|U_{\text {exact }}(t)-U_{\mathrm{hp}}(t)\right\|_{2}}{\left\|U_{\text {exact }}(t)\right\|_{2}}=\left\{\frac{\sum_{n}\left(U_{\text {exact }}\left(t^{n}\right)-U_{\mathrm{hp}}\left(t^{n}\right)\right)^{2}}{\sum_{n}\left(U_{\text {exact }}\left(t^{n}\right)\right)^{2}}\right\}^{1 / 2}
$$

This quantity can serve to tell how well the GFEM and exact curves for internal energy versus time match up.

\subsection{GFEM Simulations Using Special Enrichment Functions}

In this section we present results for simulations of the model problem using special, exponential enrichment functions. The set of enrichment functions applied to nodes whose support intersect the spike at $x=x_{0}$ is

$$
L_{\alpha i}=\left\{1, \frac{x-x_{\alpha}}{h_{\alpha}}, \exp ^{-\left(x-x_{0}\right)^{2}}\right\}
$$

where $x_{\alpha}$ is the $x$-coordinate of the node and $h_{\alpha}$ is a scaling parameter equal to the size of the largest element sharing the node [15, 31]. The resulting GFEM shape functions built using (5) and (47) are not time-dependent. Nodes whose support do not intersect the spike are enriched with

$$
L_{\alpha i}=\left\{1, \frac{x-x_{\alpha}}{h_{\alpha}}\right\}
$$

which leads to quadratic GFEM shape functions in the $x$-direction.

For the 1-D discretizations, a coarse mesh consisting of 5, $100 \mathrm{~mm}$ long elements and a fine mesh with 200, 
$2.5 \mathrm{~mm}$ long elements are used. A comparison of element sizes for these meshes is shown in Figure 3. The coarse mesh uses enrichments (47) and has only 14 degrees of freedom while the fine mesh uses (only) enrichments (48) and has 402 degrees of freedom.

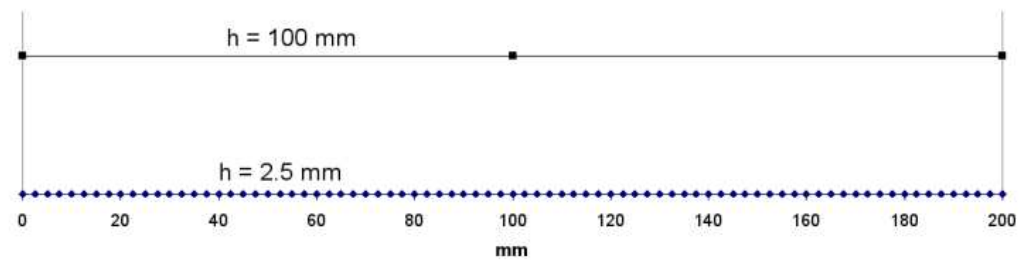

Figure 3: Comparison of element sizes for 1-D meshes with and without exponential enrichment.

For the 2-D discretizations, a coarse and a fine mesh with elements of length $6 \mathrm{~mm}$ and $2.5 \mathrm{~mm}$ in the $x$-direction, respectively, are used. In the 3-D case, the coarse (fine) mesh has elements $6 \mathrm{~mm}(3.125 \mathrm{~mm})$ in the $x$-direction near the spike, but $20 \mathrm{~mm}$ in the $x$-direction in regions far from the spike in order to save some computational effort. Nodes of the 2-D and 3-D coarse meshes whose support intersect the thermal spike are enriched with functions (47) while nodes of the fine meshes uses (only) enrichments (48).

The simulation results are compared in Table 1 in order to illustrate the benefit of the special enrichments in terms of accuracy and efficiency. As can be seen in the table, there is a significant reduction in CPU Time, as well as in the relative error, $L_{2}^{\text {error }}(U(t))$, for the meshes that use exponential enrichments, even though the elements are larger than in the meshes without exponential enrichments. It should also be noted that the size of the elements in the 2- and 3-D discretizations with exponential enrichments is restricted mainly due to our ability to accurately integrate the sharply varying source terms, as well as the exponential term in the shape functions. Internal energy versus time curves are plotted in Figure 4 for 1-, 2- and 3-D simulations.

Table 1: Comparison of output for discretizations with and without exponential enrichment functions. In the table, $h_{x}$ stands for element size in the $x$-direction.

\begin{tabular}{ccccc}
\hline Exponential Enrich. & $h_{x}(\mathrm{~mm})$ & $L_{2}^{\text {error }}(\mathrm{U}(\mathrm{t}))$ & CPU Time $(\mathrm{sec})$ & Dimension \\
\hline Yes & 100 & $5.58 \mathrm{e}-5$ & 0.0203 & $1-\mathrm{D}$ \\
No & 2.5 & 0.1979 & 4.5097 & $1-\mathrm{D}$ \\
\hline Yes & 6 & 0.0019 & 1.640 & $2-\mathrm{D}$ \\
No & 2.5 & 0.1974 & 15.580 & $2-\mathrm{D}$ \\
\hline Yes & 6 & 0.0076 & 1.230 & $3-\mathrm{D}$ \\
No & 3.125 & 0.1874 & 10.455 & $3-\mathrm{D}$ \\
\hline
\end{tabular}

\subsubsection{Effect of Volumetric Heat Capacity Magnitude}

The previous results correspond to a volumetric heat capacity $\rho c=(\pi / L)^{2}$, yielding a value of $\rho c=3.9 e-5$ for the model used here. With a very small value of $\rho c$ the transient effects are kept to a minimum. In this section, the effect of larger values of $\rho c$ on the accuracy of the internal energy is investigated. Figure 5 shows the internal energy versus time curves for discretizations using exponential enrichments, for larger values of $\rho c$. Table 2 summarizes the relative error, $L_{2}^{\text {error }}(U(t))$, obtained for larger values of $\rho c$ for discretizations with and without the use of the exponential enrichment functions. For each value of $\rho c$ investigated it is seen that the addition of the special, exponential enrichment function greatly improves the error levels by 


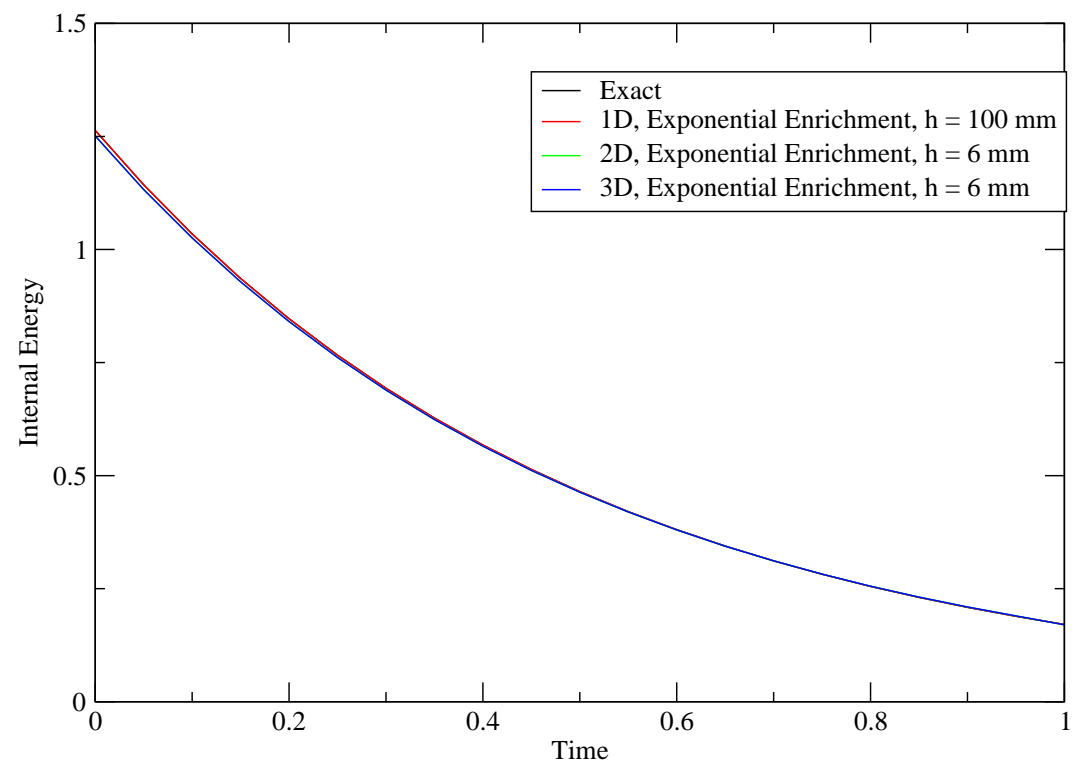

Figure 4: Plot of internal energy versus time for the 1-, 2-, and 3-D discretizations with exponential enrichments.

inserting the necessary information into the solution space, enabling a high degree of accuracy on a relatively coarse mesh. The accuracy, however, decreases as $\rho c$, and the transient effects, increase.

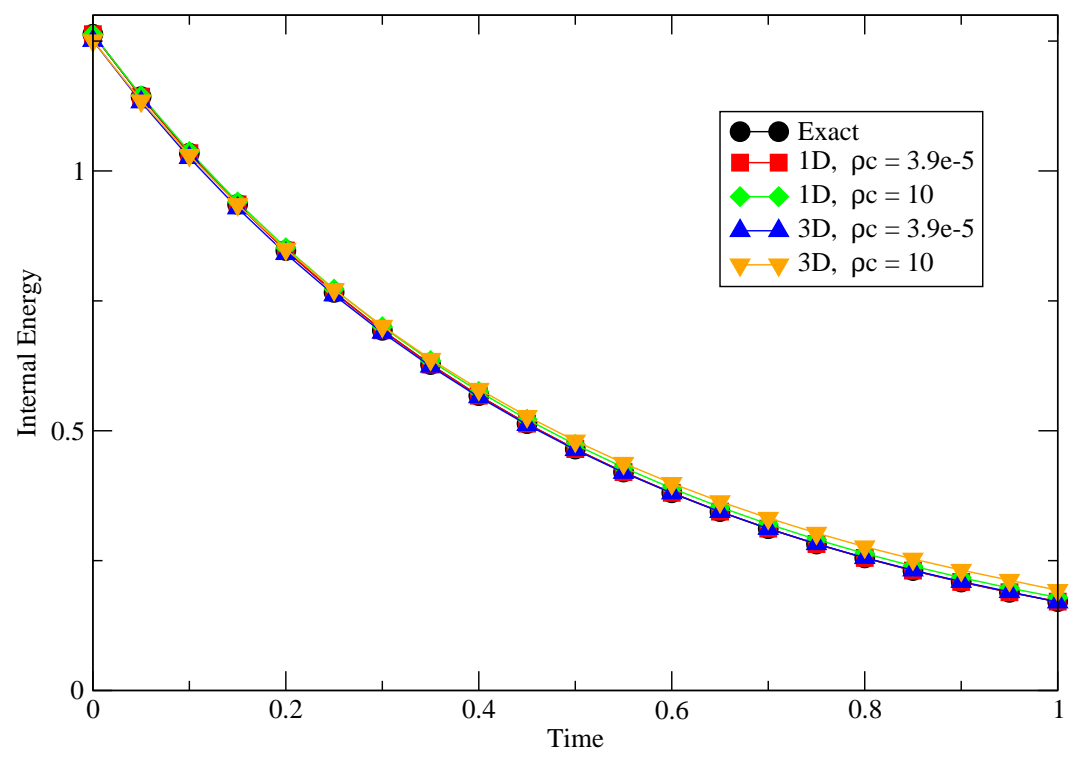

Figure 5: Internal energy versus time curves generated with exponential enrichment functions and increasing values of volumetric heat capacity . 
Table 2: Effect of volumetric heat capacity magnitude.

\begin{tabular}{cccc}
\hline Exponential Enrich. & $L_{2}^{\text {error }}(U(t)), \rho c=\frac{\pi}{L}^{2}$ & $L_{2}^{\text {error }}(U(t)), \rho c=10$ & Dimension \\
\hline Yes & $5.58 \mathrm{e}-5$ & 0.0115 & $1-\mathrm{D}$ \\
No & 0.9920 & 0.9920 & $1-\mathrm{D}$ \\
\hline Yes & 0.0076 & 0.0250 & $3-\mathrm{D}$ \\
No & 0.8772 & 0.8654 & $3-\mathrm{D}$ \\
\hline
\end{tabular}

\subsection{GFEM Simulations Using Special, Time-Dependent Enrichment Functions}

In this section, the same problem as in the previous section is solved, but now a time-dependency is inserted into the exponential GFEM shape functions through the use of the following enrichment basis

$$
L_{\alpha i}=\left\{1, \frac{x-x_{\alpha}}{h_{\alpha}}, \exp ^{-\left(x-x_{0}\right)^{2}} * \exp ^{-t}\right\}
$$

With time-dependency inserted in the shape functions, it becomes important to distinguish which formulation, described in Section 4, is being used. We first investigate the use of time-dependent shape functions with Formulation 1 in which the heat equation is first discretized spatially, and then temporally (cf. Section 4.1). In other words, we seek to investigate the effect on solution accuracy of using the standard $\alpha$-method as commonly formulated with no specific modifications to accommodate for shape functions evolving in time.

Figure 6 shows the internal energy versus time curves for various values of $\rho c$ using Formulation 1 . As can be seen, as the value of $\rho c$ is increased, the changes in the capacity matrix due to the changing enrichment functions become more significant. With very small values of $\rho c$ the problem behaves similarly to solving a series of steady-state problems, with no real thermal inertial effects being evident. It is seen from the plots that the time-dependency causes a deterioration in the behavior of the algorithm for large values of $\rho c$. Therefore, Formulation 1 is not appropriate for time-dependent shape functions.

With the previous results in mind, the performance of Formulation 2 (cf. Section 4.2) is investigated. With the Transient Formulation 2, we see significantly improved behavior in the internal energy versus time curves for 1-D simulations with large values of $\rho c$, as shown in Figure 7. Table 3 shows the significant improvement of the $L_{2}^{\text {error }}(U(t))$ for each value of $\rho c$ investigated. Figure 8 shows the dramatic improvement obtained using Formulation 2 in 1- and 3-D simulations.

Table 3: Output for Elements with Time-Dependent Exponential Enrichment Functions.

\begin{tabular}{cccc}
\hline Dimension & $\rho c$ & $L_{2}^{\text {error }}(U(t))$ Form. 2 & $L_{2}^{\text {error }}(U(t))$ Form. 1 \\
\hline 1-D & 10 & 0.0115 & 0.5537 \\
1-D & 50 & 0.0121 & 0.5584 \\
1-D & 100 & 0.0122 & 0.5590 \\
\hline 3-D & 10 & 0.0250 & 0.4139 \\
\hline
\end{tabular}

From the previous analysis it is convincing that accurate results can be generated for transient simulations involving localized heat sources on coarse meshes. In general, this is only possible provided that an enrichment function with good approximation properties is available, and the proper transient formulation is 


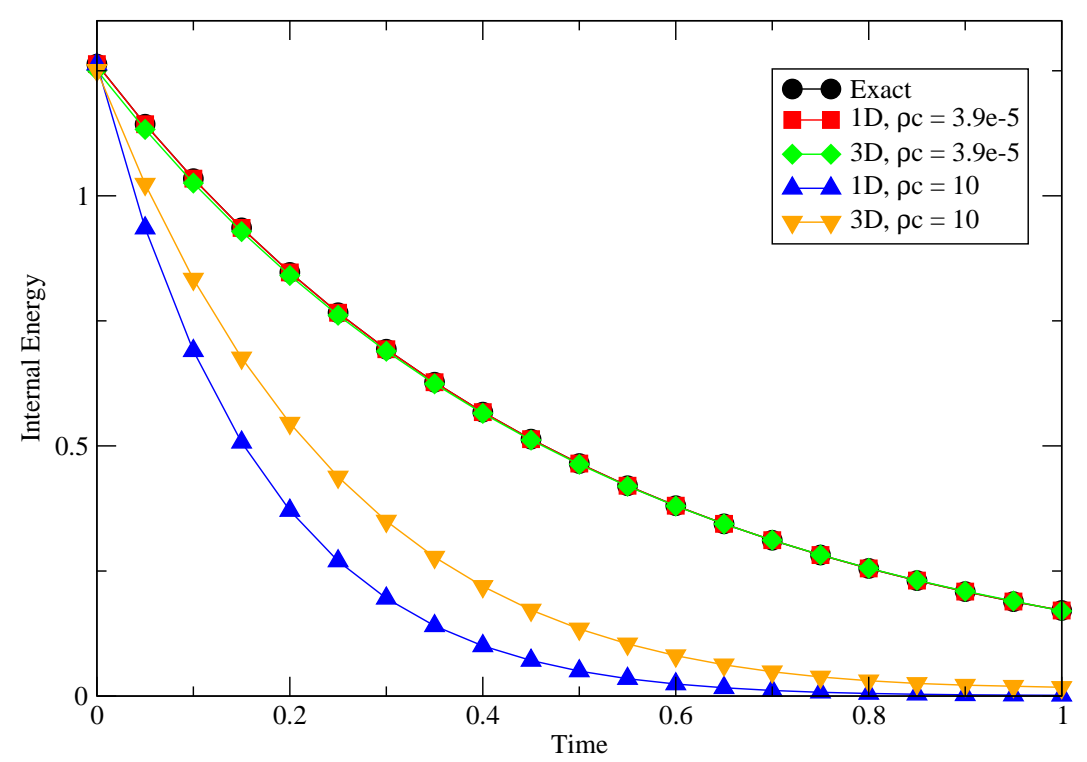

Figure 6: Internal energy versus time curves generated with 1-D and 3-D meshes enriched with time-dependent exponential enrichment functions and Formulation 1.

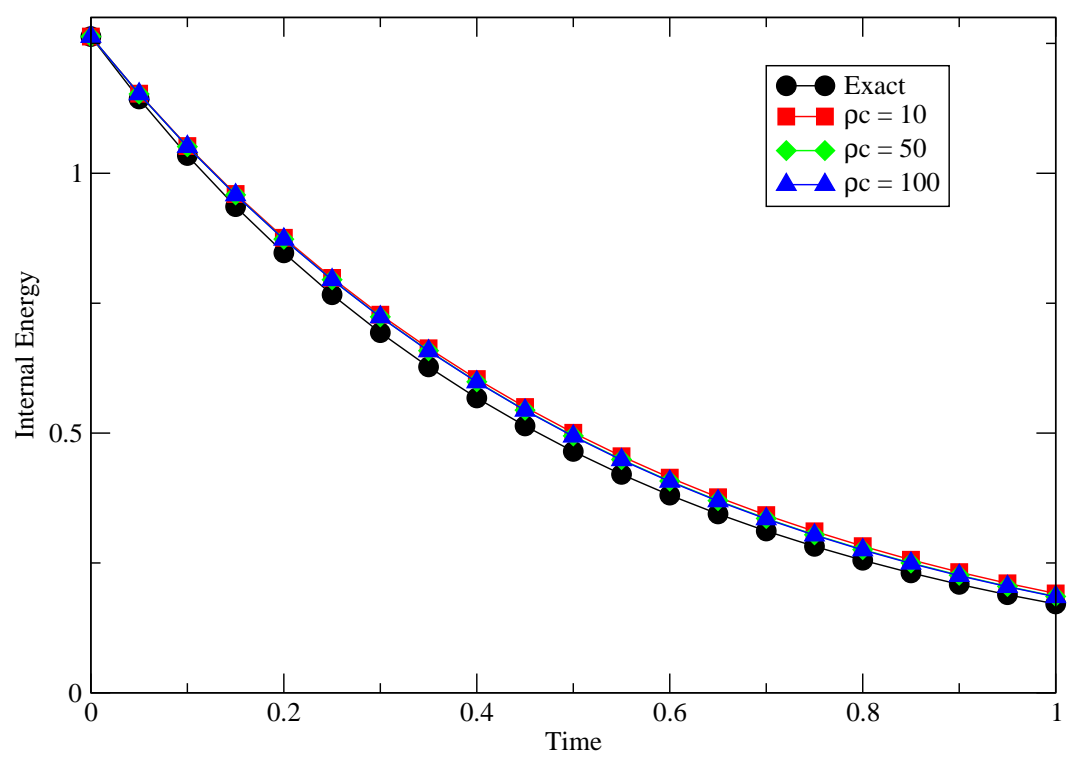

Figure 7: Internal energy versus time curves generated with 1-D time-dependent exponential enrichment functions, large values of $\rho c$, and Transient Formulation 2. 


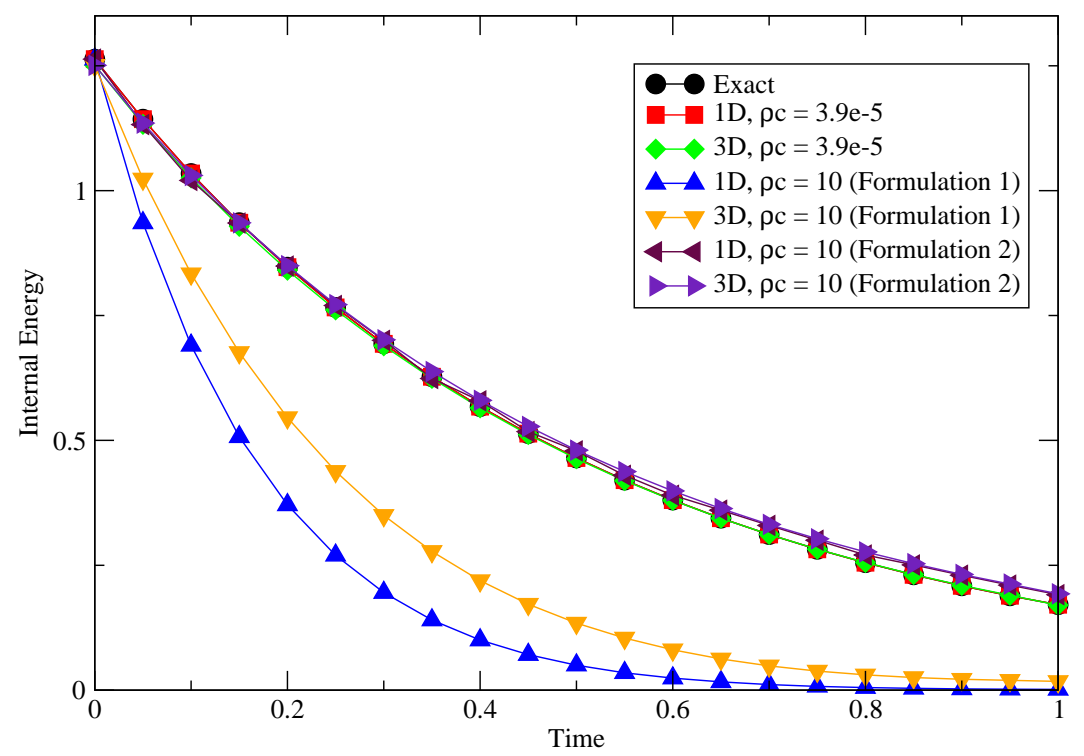

Figure 8: Internal energy versus time curves generated with 1- and 3-D time-dependent exponential enrichment functions.

used. In the general case, no such enrichment functions are known a priori. As such, we propose to generate enrichment functions on the fly via the solution of local boundary value problems as in the GFEM with global-local enrichments $\left(G F E M^{\mathrm{gl}}\right)$. The transient formulation for the $G F E M^{\mathrm{gl}}$ is provided in the next section.

\section{GFEM $^{\text {gl }}$ for Time-Dependent Problems}

In this section, we present a procedure to build enrichment functions for the class of problems governed by the heat equation (1), subjected to boundary conditions (2) and (3) and initial conditions (4). A formulation for the $G F E M^{\mathrm{gl}}$ for steady-state heat transfer, along with applications can be found in [32]. The formulation and application of the $G F E M^{\mathrm{gl}}$ to three-dimensional elasticity equations can be found in $[14,17,25]$.

In the $G F E M^{\mathrm{gl}}$ for time-dependent problems, we assume that an approximation $u_{G}^{n-1}(\boldsymbol{x})$ of the solution $u(\boldsymbol{x}, t)$ at time $t=t^{n-1}=(n-1) \Delta t$ is available. An approximation $u_{G}^{n}(\boldsymbol{x})$ of $u\left(\boldsymbol{x}, t^{n}\right)$ is computed using Formulation 2 of Section 4.2. Equation (19) with $\alpha=1.0$ leads to the following problem:

Find $u_{G}^{n} \in S_{G}^{G F E M, n}(\Omega) \subset H^{1}(\Omega)$ such that, $\forall w_{G}^{n} \in S_{G}^{G F E M, n}(\Omega)$

$$
\begin{aligned}
& \frac{\rho c}{\Delta t} \int_{\Omega} w_{G}^{n} u_{G}^{n} d \Omega+\int_{\Omega}\left(\nabla w_{G}^{n}\right)^{T} \kappa \nabla u_{G}^{n} d \Omega+\eta \int_{\Gamma_{c}} u_{G}^{n} w_{G}^{n} d \Gamma= \\
& \frac{\rho c}{\Delta t} \int_{\Omega} w_{G}^{n} u_{G}^{n-1} d \Omega+\int_{\Gamma_{f}} \bar{f}^{n} w_{G}^{n} d \Gamma+\eta \int_{\Gamma_{c}} \bar{u}^{n} w_{G}^{n} d \Gamma+\int_{\Omega} w_{G}^{n} Q^{n} d \Omega
\end{aligned}
$$

where $S_{G}^{G F E M, n}(\Omega) \subset H^{1}(\Omega)$ is the generalized FEM space at time step $n$. The enrichment functions in $S_{G}^{G F E M, n}(\Omega)$ are defined in local spaces and have to be computed; we describe a fine-scale problem in the next subsection to achieve this goal. Note that the same approximation space is used for the GFEM solution $u_{G}^{n}$ and the weight function $w_{G}^{n}$ as discussed in Section 4.2. The mesh used to solve problem (50) is typically a coarse quasi-uniform mesh, even when the solution is not smooth. Problem (50) leads to a system of linear equations for the unknown degrees of freedom of $u_{G}^{n}$. 


\subsection{Fine-Scale Problems at Time $t^{n}$}

The proposed FFEM $^{\mathrm{gl}}$ involves the solution of a fine-scale boundary value problem defined in a neighborhood $\Omega_{L}$ of thermal spikes, where strong solution gradients develop. The local domain $\Omega_{L}$ is composed of the union of clouds $\omega_{\alpha}$ of the open cover $\left\{\omega_{\alpha}\right\}_{\alpha=1}^{N}$ of $\Omega$ that intersect or are close to a thermal spike. A-posteriori error estimate measures can be used to define $\Omega_{L}$ but that is beyond the scope of this paper.

Having the global approximation $u_{G}^{n}$ at time $t^{n}$, we compute the following fine-scale problem on $\Omega_{L}$ to find enrichment functions for the space $S_{G}^{G F E M, n+1}(\Omega)$ :

Find $u_{L}^{n} \in S_{L}^{G F E M, n}\left(\Omega_{L}\right) \subset H^{1}\left(\Omega_{L}\right)$ such that, $\forall w_{L}^{n} \in S_{L}^{G F E M, n}\left(\Omega_{L}\right)$

$$
\begin{aligned}
& \int_{\Omega_{L}}\left(\nabla u_{L}^{n}\right)^{T} \kappa \nabla w_{L}^{n} d \Omega+\eta \int_{\partial \Omega_{L} \backslash\left(\partial \Omega_{L} \cap \Gamma_{f}\right)} u_{L}^{n} w_{L}^{n} d \Gamma= \\
& \eta \int_{\partial \Omega_{L} \backslash\left(\partial \Omega_{L} \cap \partial \Omega\right)} u_{G}^{n} w_{L}^{n} d \Gamma+\eta \int_{\partial \Omega_{L} \cap \Gamma_{c}} \bar{u}^{n+1} w_{L}^{n} d \Gamma+ \\
& \int_{\Omega_{L}} Q^{n+1} w_{L}^{n} d \Omega+\int_{\partial \Omega_{L} \cap \Gamma_{f}} \bar{f}^{n+1} w_{L}^{n} d \Gamma
\end{aligned}
$$

where, $S_{L}^{G F E M, n}\left(\Omega_{L}\right)$ is a discretization of $H^{1}\left(\Omega_{L}\right)$ using, e.g., standard GFEM shape functions. It is possible, however, to use other methods, like the standard FEM or the Boundary Element Method, to solve fine-scale problems. The proposed methodology enables one to select the most effective method for the particular class of fine scale problem considered. Thus, the methodology is highly flexible and general.

A key aspect of problem (51) is the use of the coarse-scale solution at time step $t^{n}, u_{G}^{n}$, as boundary condition on $\partial \Omega_{L} \backslash\left(\partial \Omega_{L} \cap \partial \Omega\right)$. In the numerical experiments presented in Section 7, the parameter $\eta$ is taken as a penalty number. Thus Dirichlet boundary conditions are enforced on $\partial \Omega_{L} \backslash\left(\partial \Omega_{L} \cap \Gamma_{f}\right)$. Exact boundary conditions are prescribed on portions of $\partial \Omega_{L}$ that intersect either $\Gamma_{c}$ or $\Gamma_{f}$. Another key point of problem (51) is that no transient effects are considered. However, the source function, $Q$, and the boundary conditions on $\partial \Omega_{L} \cap \Gamma_{c}$ and $\partial \Omega_{L} \cap \Gamma_{f}$ are computed at time step $t^{n+1}$. The rationale for this is that $u_{L}^{n}$ is used to define the global solution space at time $t^{n+1}$, as described in the section below.

\subsection{Scale-Bridging with Global-Local Enrichment Functions}

The solution, $u_{L}^{n}$, of the fine-scale problem defined above is used to build generalized FEM shape functions defined on a coarse global mesh:

$$
\phi_{\alpha i}^{n+1}(\boldsymbol{x}):=\varphi_{\alpha}(\boldsymbol{x}) u_{L}^{n}(\boldsymbol{x})
$$

where the partition of unity function, $\varphi_{\alpha}$, is provided by a global, coarse, FE mesh and $u_{L}^{n}$ has the role of an enrichment or basis function for the patch space $\chi_{\alpha}\left(\omega_{\alpha}\right)$. Hereafter, $u_{L}^{n}$ is denoted a global-local enrichment function. The global GFEM space containing shape functions $\phi_{\alpha i}^{n+1}$ is denoted $S_{G}^{G F E M, n+1}(\Omega)$. The coarse scale problem (50) is solved for $u_{G}^{n+1} \in S_{G}^{G F E M, n+1}(\Omega)$ and the procedure is repeated at each time step. The $G F E M^{\mathrm{gl}}$ for time-dependent problems is illustrated in Figure 9. The global solution provides boundary conditions for fine-scale problems while local solutions are used as enrichment functions for the coarse problem through the partition of unity framework of the GFEM.

Let us point out that only a few degrees of freedom are added to the global (coarse-scale) discretization even if the computation of the fine-scale solution requires several thousands of degrees of freedom since $u_{L}^{n}$ is a known function at time step $t^{n+1}$. The global problem is solved on the coarse global mesh enriched with the shape functions defined in (52). These functions are hierarchically added to the FE discretization, and thus, a few entries are added to element matrices while keeping existing ones associated with standard FE 


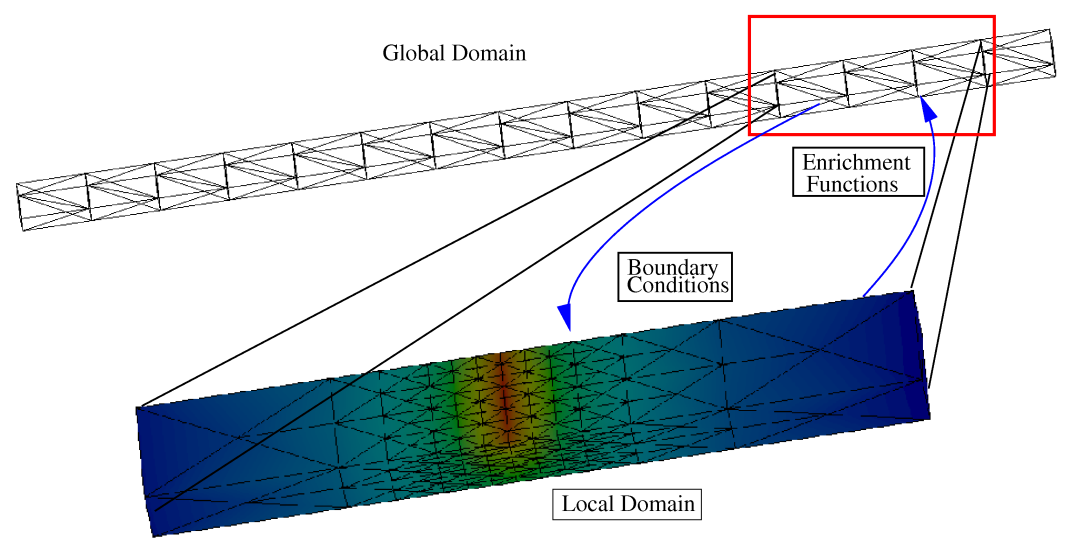

Figure 9: Illustration of the $\mathrm{GFEM}^{\text {gl }}$ for time-dependent problems. The global domain is discretized with a coarse mesh, even if the solution is not smooth. The coarse-scale problem at $t^{n}$ provides boundary conditions for local problems containing sharp thermal spikes. The solution of these problems are used to build approximation spaces for the coarse-scale problem at time $t^{n+1}$.

shape functions. The hierarchical nature of the global-local enrichments can be exploited in the solution of the global problem and avoid the solution of the problem from scratch at every time step. This is in contrast with available adaptive finite element methods.

Iterative Improvement of Global-Local Enrichment Functions A key feature of the methodology described above is the use of available information at a simulation step $t^{n}$ to build the solution space for the next time step, i.e., the GFEM space $S_{G}^{G F E M, n+1}(\Omega)$ containing the GFEM solution $u_{G}^{n+1}$. The coarse-scale solution at time step $t^{n}, u_{G}^{n}$, is used as boundary condition on $\partial \Omega_{L} \backslash\left(\partial \Omega_{L} \cap \partial \Omega_{G}\right)$ for the fine-scale problem (51) instead of the unknown exact solution at time $t^{n+1}$. As a result, the error of $u_{L}^{n}$ depends not only on the discretization used in the local domain $\Omega_{L}$, but, also on how much the solution of the problem changes at $\partial \Omega_{L} \backslash\left(\partial \Omega_{L} \cap \partial \Omega_{G}\right)$ between time steps. The effect of the inexact boundary conditions on the accuracy of $u_{L}^{n}$ can be addressed by repeating the above procedure at each time step:

(i) Use the solution of the global problem $u_{G}^{n+1} \in S_{G}^{G F E M, n+1}(\Omega)$ as boundary conditions for the fine-scale problem (51) at time $t^{n}$;

(ii) update global shape functions (52) and global solution space $S_{G}^{G F E M, n+1}(\Omega)$.

(iii) solve the coarse scale problem (50) for $u_{G}^{n+1} \in S_{G}^{G F E M, n+1}(\Omega)$.

(iv) Go to step (i) if the accuracy of $u_{G}^{n+1}$ is not acceptable; proceed to the next time step otherwise.

In Section 7.2.1, the effect of time-step size on the accuracy of the $G F E M^{\mathrm{gl}}$ is investigated.

The performance of the GFEM $^{\mathrm{gl}}$ when solving transient heat transfer problems with solutions exhibiting highly localized sharp thermal gradients is investigated in the next section. As a note, a quasi-static solution is obtained at time $t^{0}$ to enforce the initial conditions (41). This is simply the solution of Poisson's equation since no time-dependency is required. Again, more details of the formulation for steady-state $G F E M^{\mathrm{gl}}$ analysis can be found in [32]. 


\section{Numerical Experiments Using GFEM $^{\mathrm{gl}}$}

\subsection{Numerical Experiment 1: Model Problem Utilizing GFEM $^{\mathrm{gl}}$ in 3-D}

In this section we apply the transient $G_{F E M^{\mathrm{gl}}}$ to the model problem as posed in Section 5. We use the same mesh as was used for the simulations with exponential enrichment functions, i.e. elements with a width of $6 \mathrm{~mm}$ in the $x$-direction. With this approach we can be assured that the only difference between the two analyses is the actual shape function itself, whether it be analytic or numerically generated. The local domain, in this instance, is selected to be the entire domain, a very poor choice in the general case, but it ensures the use of exact Neumann boundary conditions in the local domain, free of any potential numerical pollution. The goal of this example is to verify Transient Formulation 2 with the GFEM ${ }^{\mathrm{gl}}$. The local domains are subjected to $h$-extensions, in which high levels of refinement are used only locally in the region of the spike, resulting in highly graded local meshes with a uniform, orthotropic polynomial order of $\left(p_{x}, p_{y}, p_{z}\right)=(4,1,1)$.

Figure 10 shows global internal energy, $U(t)$, versus time curves. In Figure 10, as well as subsequent figures, one level of mesh refinement indicates one localized mesh refinement cycle in which the markededge algorithm $[1,7]$ is used for the bisection of the tetrahedral elements. The volumetric heat capacity, $\rho c$, is taken as $3.9 e-5$. Transient Formulations 1 and 2 provide nearly identical results in this case and only curves computed with Formulation 1 are shown. The effect of adding global-local enrichment functions is significant. The figure shows that the error on $U(t)$ can be controlled through mesh refinement in the local problem, thus avoiding refinement of the global mesh even when no a-priori knowledge about the exact solution is used.

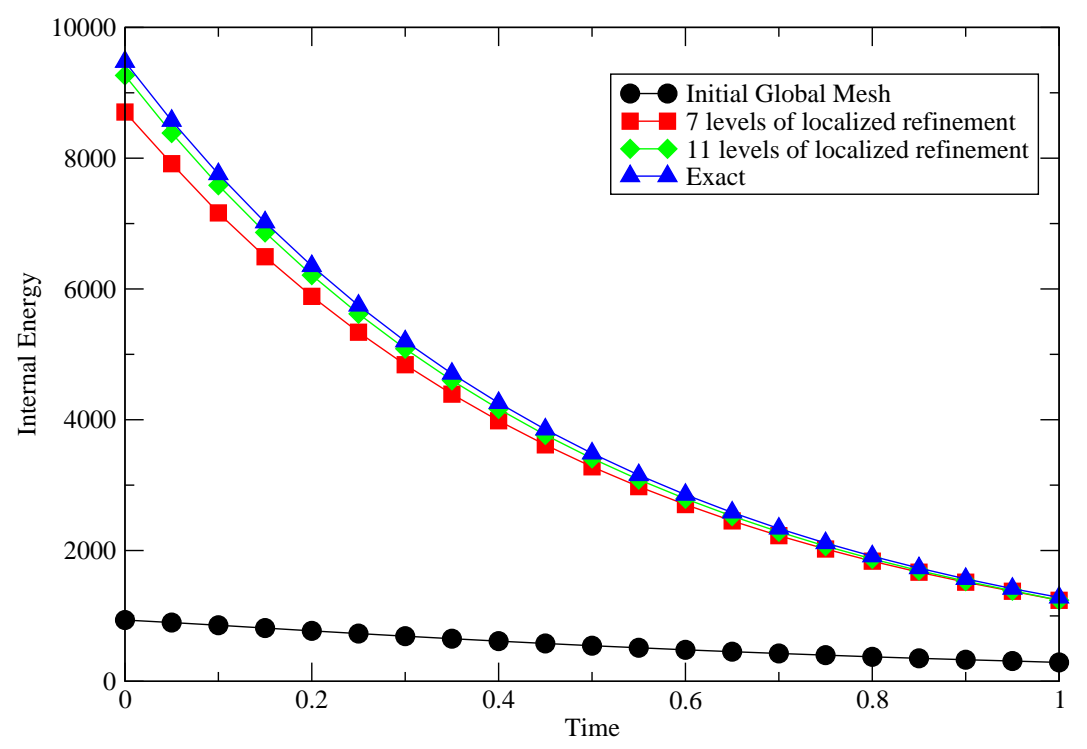

Figure 10: Plots of internal energy versus time for $\mathrm{GFEM}^{\text {gl }}$ solutions with increasing levels of mesh refinement in local problem. The volumetric heat capacity is taken as $\rho c=3.9 e-5$. All curves computed with Transient Formulation 1.

As was the case in Section 5.2, we now increase the value of $\rho c$ and analyze its impact on the behavior of the solution. As is shown in Figure 11, we again see that as the value of $\rho c$ is increased, the quality of results provided by Transient Formulation 1 deteriorates because of the time-dependency of the shape functions used in the $G F E M^{\mathrm{gl}}$. Results obtained from FFEM $^{\mathrm{gl}}$ simulations and Transient Formulation 2 are also shown. From the curve corresponding to Formulation 2, it can be seen that the time-dependency of the shape functions are once again properly accounted for, yielding much more accurate results. The relative 
error in internal energy for Formulations 1 and 2 are $L_{2}^{\text {Error }}(U(t))=0.4266$ and $L_{2}^{\text {Error }}(U(t))=0.0271$, respectively, when $\rho c=10$.

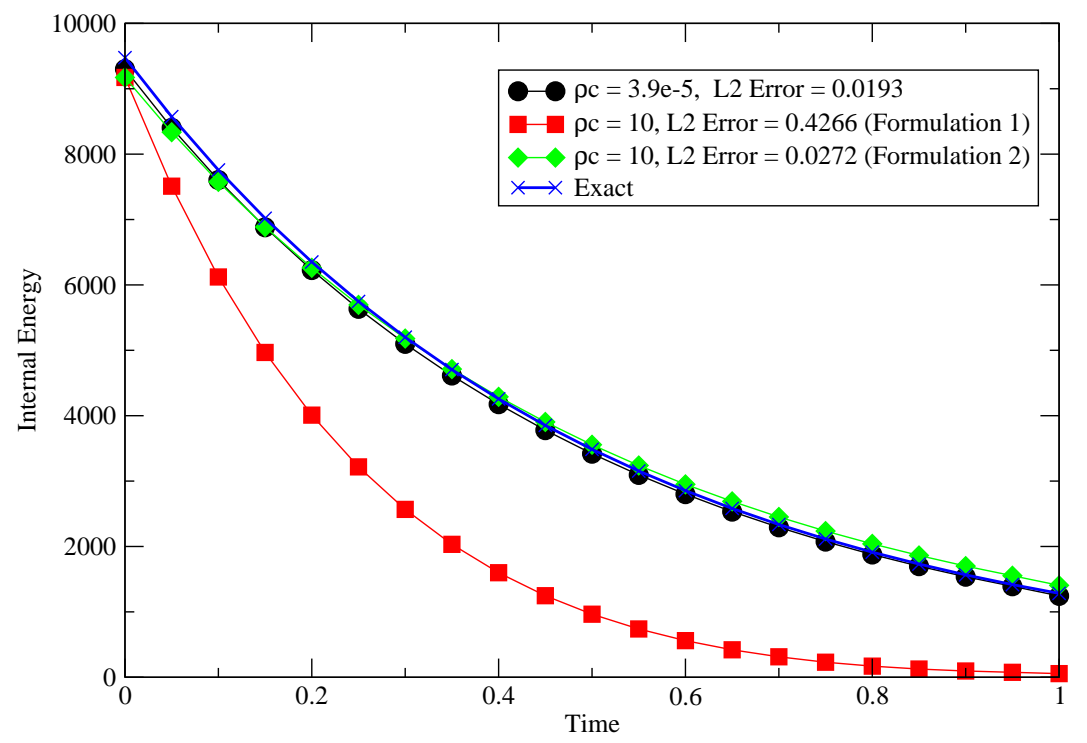

Figure 11: Plot of internal energy versus time for GFEM ${ }^{g l}$ solutions in 3-D for $\rho c=3.9 e-5$ and $\rho c=10$. For $\rho c=10$ both transient formulations are investigated. The curve for the exact solution is given by (39) and (43), and is independent of $\rho c$. 


\subsection{Numerical Experiment 2: Beam Subjected to Stationary Laser Flux}

In this section, the transient $G F E M^{\mathrm{gl}}$ is applied to a beam subjected to a normal, surface flux. We apply the methodology first to an Aluminum ( $\mathrm{Al}$ ) beam, and then to a Silicon Carbide $(\mathrm{SiC}$ ) beam, both subjected to a Gaussian laser flux (53), the shape of which is shown in Figure 12.

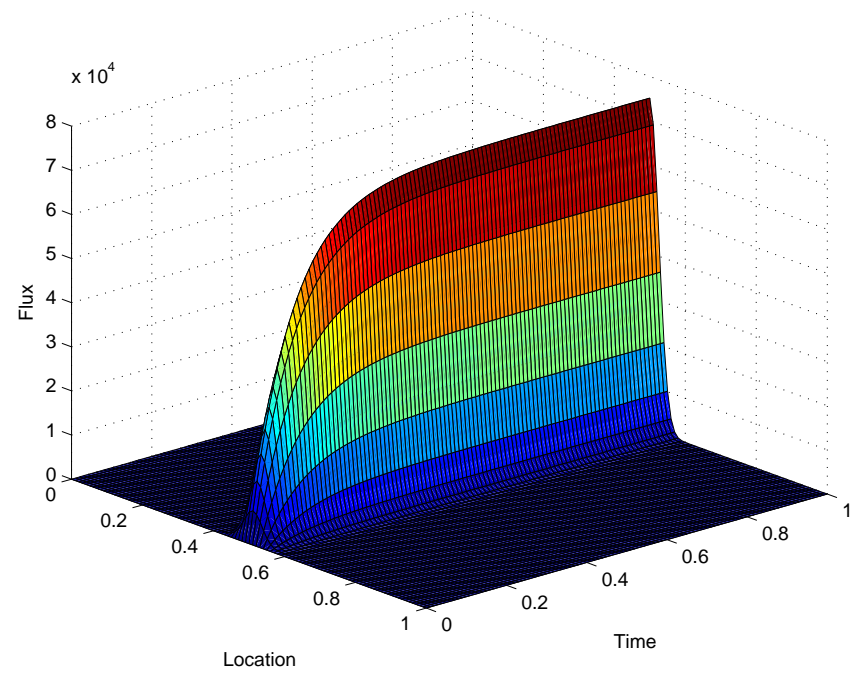

Figure 12: Spatial and temporal variation of the Gaussian laser $(\gamma=10)$.

The applied Gaussian laser flux is modeled as in [10], taking the form:

$$
\begin{gathered}
\bar{f}(\boldsymbol{x}, t)=I_{0} * f(t) * \frac{1}{2 \pi a^{2}} * G(\boldsymbol{x}, b, a) \\
f(t)=1-\exp (-\gamma * t) \\
G(\boldsymbol{x}, b, a)=\exp \left(\frac{-(x-b)^{2}}{2 a^{2}}\right)
\end{gathered}
$$

In the above equations, the constants take the values: $I_{0}=295 \frac{f t-l b f}{s}, a=0.025 \mathrm{in}, \gamma=10.0 \mathrm{~s}^{-1}, b=9.3 \mathrm{in}$. From this analysis we seek to determine the effect of the value of $\rho c$ for simulations in which the beam has material parameters similar to those of an actual engineering material. Table 4 shows the values used for the material parameters in the numerical simulations.

Table 4: Material Parameters.

\begin{tabular}{ccc}
\hline Material & $\kappa\left(\frac{f t-l b f}{\operatorname{s} \cdot \mathrm{in}^{\circ} \mathrm{F}}\right)$ & $\rho c\left(\frac{f t-l b f}{i n^{3} \cdot{ }^{\circ} \mathrm{F}}\right)$ \\
\hline$A l$ & 2.92 & 18.3 \\
$\mathrm{SiC}$ & 1.32 & 15.6 \\
\hline
\end{tabular}

The beam itself is of dimension $12 \times 0.5 \times 0.24$ inches, in the $x-, y$ - and $z$-direction, respectively, and the global mesh is shown in Figure 13. Flux boundary conditions given by (53) are applied to a portion of 
the top surface of the beam at $8.0 \leq x \leq 10.0,0 \leq y \leq 0.5, z=0.24$, as noted in the figure. The rest of the boundary is subjected to convection boundary conditions (2), with $\eta=11 \frac{\mathrm{ft}-l \mathrm{lbf}}{\mathrm{s} \cdot \mathrm{in}^{2} \cdot{ }^{\circ} \mathrm{F}}$ and $\bar{u}=70^{\circ} \mathrm{F}$. In this example we seek to simulate a situation in which the applied loading evolves to a steady-state localized, sharp, surface flux as time progresses.

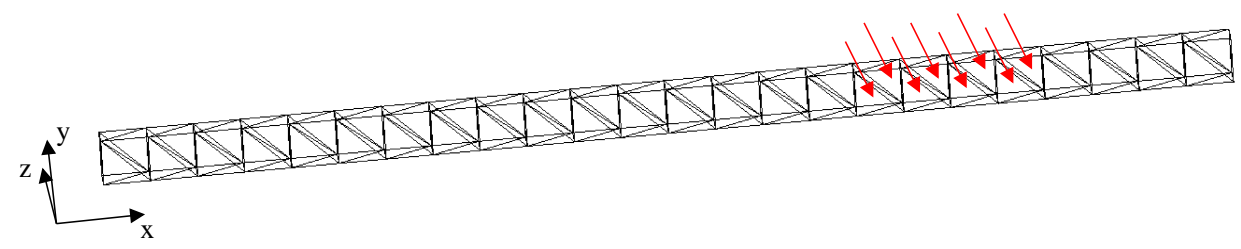

Figure 13: Global mesh used for the beam model. Flux boundary conditions are denoted by red arrows.

Steady-State Convergence Analysis We first analyze the steady-state version of the problem described above. In this case we apply the maximum value of the laser flux (i.e., the value of $\bar{f}(\boldsymbol{x}, t)$ when $t \rightarrow \infty$ ), and solve Poisson's equation for steady-state heat conduction.

A reference internal energy value is obtained using a sequence of six $h p-G F E M$ discretizations. The $h p$ GFEM is based on polynomial enrichments only and mesh refinement. Let the relative difference in internal energy between two successive solutions, say $u_{h p}^{m-1}$ and $u_{h p}^{m}$, be given by

$$
U^{\text {Diff,m }}:=\frac{\left|U_{h p}^{m}-U_{h p}^{m-1}\right|}{\left|U_{h p}^{m}\right|}
$$

where $U_{h p}^{m-1}$ and $U_{h p}^{m}$ are the internal energy of the $h p$-GFEM solutions $u_{h p}^{m-1}$ and $u_{h p}^{m}$, respectively.

Figure 14 shows the relative difference in internal energy, $U^{\text {Diff,m }}$, versus problem size of discretization $m$. The last discretization in the sequence, $u_{h p}^{m=5}$, has 821,412 dofs, a uniform polynomial order of $p=3$, and $U^{\text {Diff,m=5 }}=\mathscr{O}\left(10^{-6}\right)$. Based on these results, the reference internal energy is taken as $U_{r e f}=U_{h p}^{m=5}=$ $2.8575 e 6$.

Figure 15 shows the relative error in the energy norm for $h p$-GFEM and GFEM ${ }^{\mathrm{gl}}$ solutions. In the case of the FFEM $^{\mathrm{gl}}$ the horizontal axis shows the element size in the local domain. The global mesh is the one shown in Figure 13. As a result, the $G_{F E M^{\mathrm{gl}}}$ discretization has 1,020 dofs, regardless of the local problem size. In contrast, the number of dofs in the $h p-G F E M$ is in the range $[1,000-190,000]$. For this problem, only one global-local iteration is required, as the boundary conditions in the local domain are sufficiently accurate, and a second iteration did not appreciably improve the error level achieved. It may be noted that in all numerical examples presented in this paper, no iterations between global and local problems aimed at improving the enrichment functions are required. Based on the results presented in [32] it is reasonable to assume that if additional iterations are required, it is likely that they will only be needed at the first time step to take care of numerical pollution effects. No iterations to select the local domain size are performed either. In fact, as demonstrated in [33], increasing the size of the local domain does not necessarily improve the quality of enrichment functions for the class of problems considered in this paper.

As was the case with the $h p-G F E M$, a uniform polynomial order of $p=3$ is used in the GFEM ${ }^{\mathrm{gl}}$ analysis. As can be seen from the plot, the $h p$-GFEM achieves a convergence rate of $\beta=3.07$, as compared to the optimum convergence rate of $\beta_{\text {opt }}=3.0$. The $G F E M^{\mathrm{gl}}$ achieves a slightly lower, yet comparable convergence rate of $\beta=2.61$. In both cases, a sufficiently refined mesh must be used in order to approach optimal convergence rates. This is due to the roughness of the solution. 


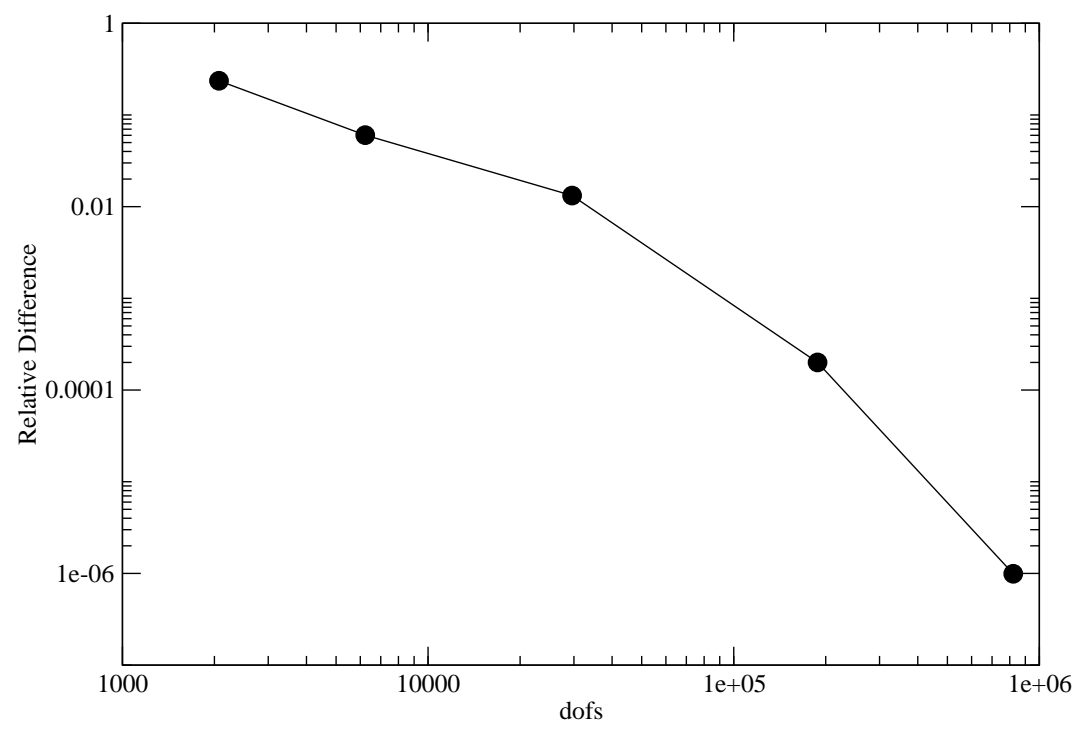

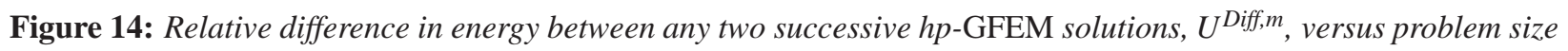
of discretization m. Analysis performed to determine reference value for steady-state internal energy.

In the discretizations used in this paper, the $h p$-GFEM solution spaces contain the global-local enrichment functions used in the definition of $G F E M^{\mathrm{gl}}$ solution spaces. However, most of the enrichment functions used in the definition of $h p$-GFEM spaces do not belong to $G F E M^{\mathrm{gl}}$ solution spaces. As a result, the convergence rate of the $G F E M^{\mathrm{gl}}$ may be lower than the one in the $h p$-GFEM when the same element sizes are used in the local problems and in the $h p$-GFEM discretizations. Figure 15 shows a representative behavior of these methods. Not only the convergence rate but also the error in the energy norm of the $G F E M^{\mathrm{gl}}$ is comparable to the $h p$-GFEM when the same element size and polynomial order are used in the hp-GFEM and in the local problem for the FFEM $^{\mathrm{gl}}$.

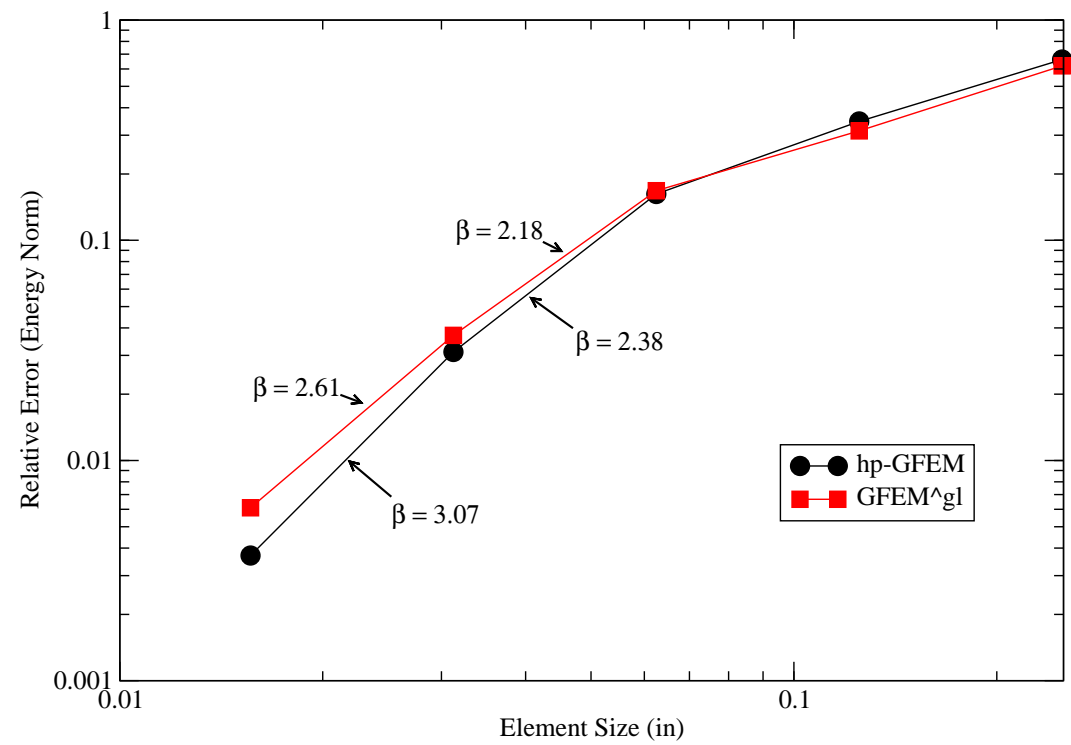

Figure 15: Convergence of the relative error in the energy norm. Convergence rates of $\beta=3.07$ and $\beta=2.61$ are obtained for $h p$-GFEM and $\mathrm{GFEM}^{g l}$ analyzes, respectively. 
Transient Analysis: Determination of Reference Solution A reference solution for the transient case is obtained using the same approach as in the steady-state case. Here, the hp-GFEM discretizations use high levels of local refinement and a non-uniform, non-isotropic $p$-enrichment strategy in which the entire global domain has a $p$-order of $\left(p_{x}, p_{y}, p_{z}\right)=(3,3,3)$ with a local region around the laser flux with $\left(p_{x}, p_{y}, p_{z}\right)=$ $(4,3,4)$. The relative difference in the $L_{2}$ norm of the internal energy between two successive solutions, say $u_{h p}^{m-1}(t)$ and $u_{h p}^{m}(t)$, is computed using

$$
L_{2}^{\text {Diff,m }}(U(t))=\frac{\left\|U_{h p}^{m}(t)-U_{h p}^{m-1}(t)\right\|_{2}}{\left\|U_{h p}^{m}(t)\right\|_{2}}
$$

where $U_{h p}^{m-1}(t)$ and $U_{h p}^{m}(t)$ are the internal energy of the $h p$-GFEM solutions $u_{h p}^{m-1}(t)$ and $u_{h p}^{m}(t)$, respectively, and the discrete $L_{2}$ norm is defined in (45).

Figure 16 shows the relative difference in the $L_{2}$ norm of the internal energy, $L_{2}^{\text {Diff,m }}(U(t))$, versus problem size of discretization $m$. The last discretization in the sequence, $u_{h p}^{m=7}$, has 359,003 dofs and $L_{2}^{\text {Diff,m=7 }}(U(t))=$ $\mathscr{O}\left(10^{-7}\right)$. Based on these results, the reference internal energy is taken as $U_{r e f}(t)=U_{h p}^{m=7}(t)$.

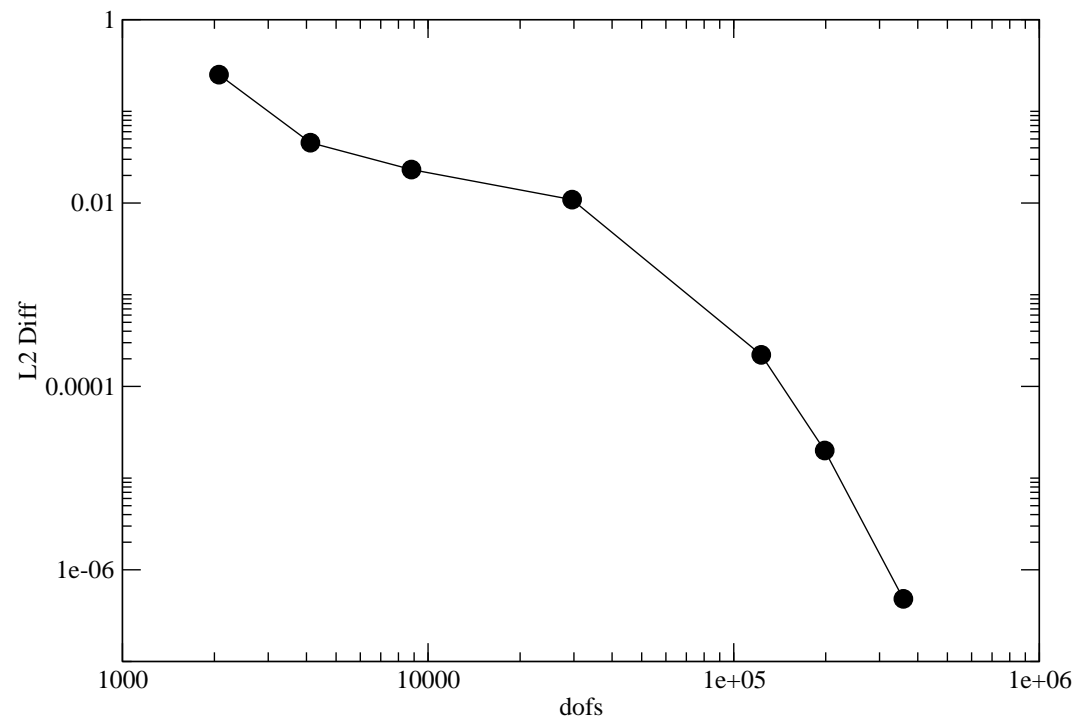

Figure 16: Relative difference in the discrete $L_{2}$ norm of the internal energy, $L_{2}^{\text {Diff, } m}(U(t))$, versus problem size of discretization $\mathrm{m}$. Solutions are computed using the $\mathrm{hp}$-GFEM.

With a reference curve for the internal energy defined, we now analyze the $A l$ beam. All of the results presented from this point forward are generated using Transient Formulation 2 since the shape functions of the FFEM $^{\mathrm{gl}}$ are time-dependent. The GFEM $^{\mathrm{gl}}$ solutions are generated using meshes with only 1,020 dofs and solution accuracy is improved through the use of $h$-extensions in the local domain. Figure 17 shows local meshes with increasing levels of refinement around the laser flux. The local domains are generated as described in [32], with seed nodes selected from a bounding box from $\min =[8.2,0.0,0.0]$ to $\max$ $=[10.6,0.5,0.24]$, and localized refinement used in a bounding box from $\min =[8.7,0.0,0.0]$ to $\max$ $=[9.7,0.5,0.24]$. It may be noted that the dimension of the local domain remains unchanged, and merely the levels of localized refinement is increased.

Results obtained for the $A l$ beam generated with the $G F E M^{\mathrm{gl}}$ are plotted in Figure 18. This figure shows that the internal energy versus time curves converge to the proper reference curve as the global-local enrichments are improved through mesh refinement in the local domain. From the figure, it is also apparent that the time- 


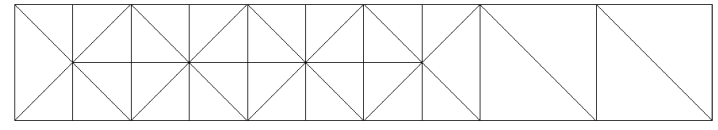

(a) 3 levels of local mesh refinement.

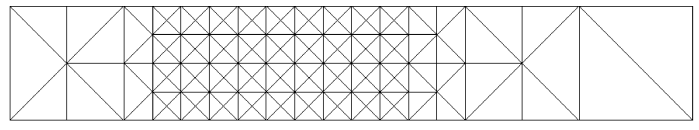

(c) 7 levels of local mesh refinement.

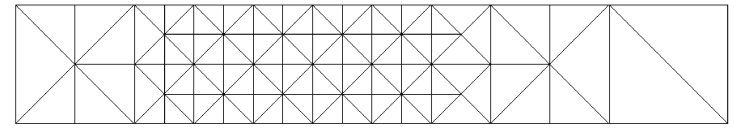

(b) 5 levels of local mesh refinement.

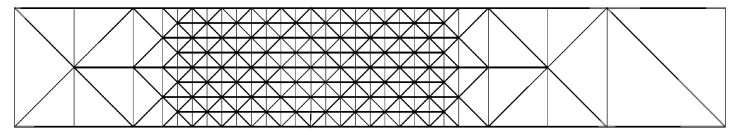

(d) 9 levels of local mesh refinement.

Figure 17: Local meshes used for $\mathrm{GFEM}^{\text {gl }}$ simulations, increasing levels of local refinement are used.

dependency of the enrichment functions is properly accounted for.

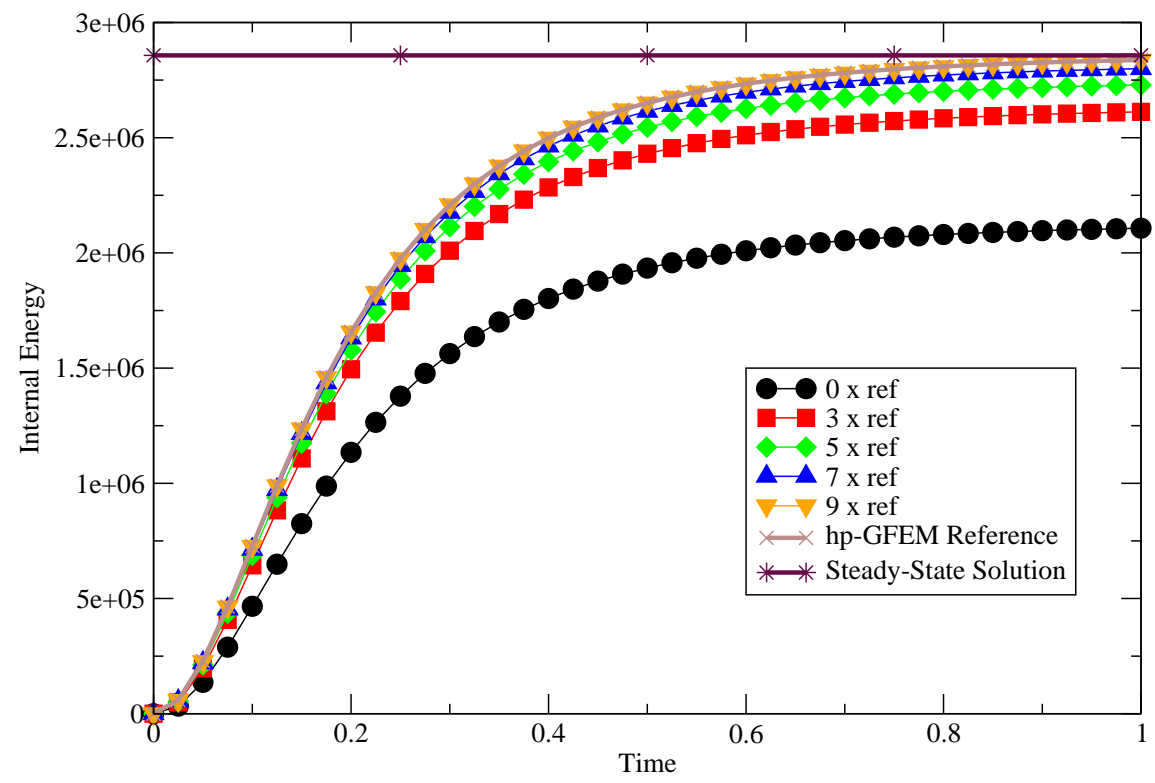

Figure 18: Internal energy versus time curves for Al. Solutions computed with $\mathrm{GFEM}^{\text {gl }}$ on a fixed global mesh and h-extensions in the local domain.

Figure 19 shows the convergence in the $L_{2}^{\text {error }}$ norm of the internal energy for the GFEM ${ }^{\mathrm{gl}}$ and $h p-G F E M$. The error norm $L_{2}^{\text {error }}$ is computed using (46) and the reference internal energy $U_{r e f}(t)$. It should be noted that in the case of the $h p$-GFEM, the shape functions are not time-dependent. The measure of computational effort is taken as the "element size", referring to the width in the $x$-direction, $h_{x}$, of the elements in the region of high refinement. For the hp-GFEM "element size" of course refers to elements in the global domain, while for the GFEM ${ }^{\mathrm{gl}}$ "element size" refers to the width of the elements used in the local problems, because the width of the elements in the global domain remains constant, $h_{x}=0.5 \mathrm{in}$. As can be seen from the plot, at a given element size, the $h p$-GFEM produces solutions with slightly better accuracy, but it does so at the increase in the number of dofs used in the simulation. The number of dofs in the hp-GFEM is in the range $[10,000-30,000]$ while the $G F E M^{\mathrm{gl}}$ discretization has 1,020 dofs. The convergence rates, $\beta$, and error levels are very comparable for both methods. This behavior is very similar to the steady-state case shown in Figure 15. The $p$-enrichment strategy for the $h p$-GFEM is $\left(p_{x}, p_{y}, p_{z}\right)=(3,3,3)$ globally, with a local 
region around $x=b$ where $\left(p_{x}, p_{y}, p_{z}\right)=(4,3,3)$. For the $\operatorname{GFEM}^{\mathrm{gl}}$ simulations $\left(p_{x}, p_{y}, p_{z}\right)=(3,3,3)$ in the global domain, and $\left(p_{x}, p_{y}, p_{z}\right)=(4,2,2)$ in the local domain.

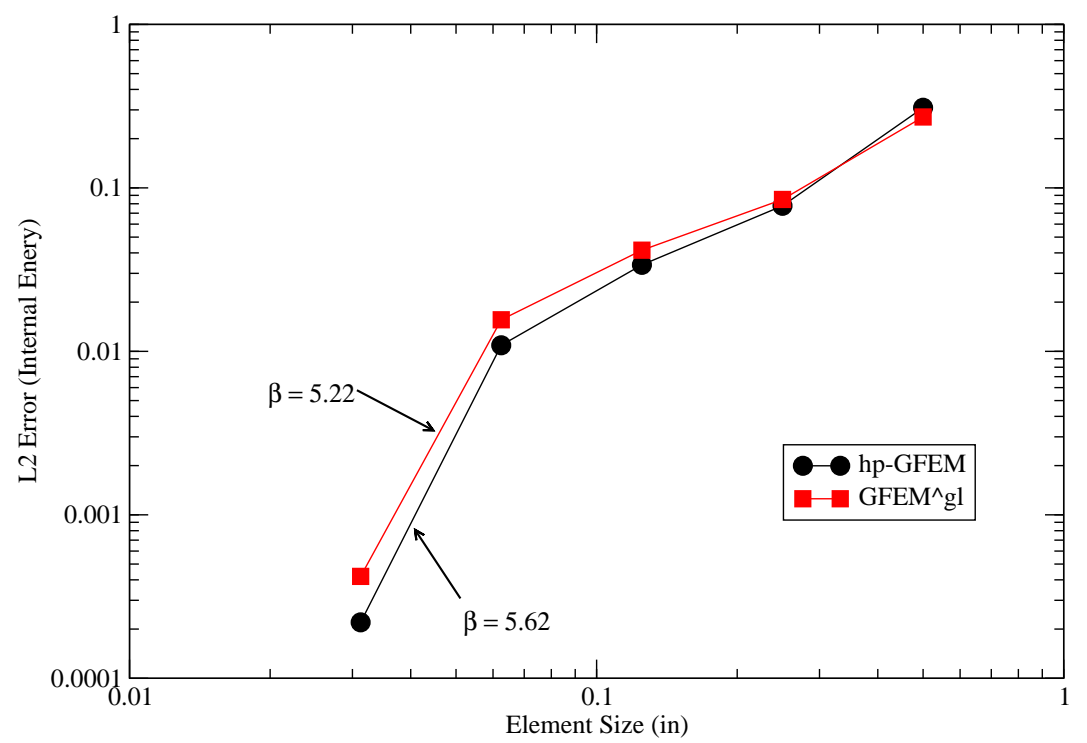

Figure 19: Convergence in $L_{2}^{\text {error }}(U(t))$ for internal energy versus time curves generated with hp-GFEM and $\mathrm{GFEM}^{g l}$.

Figures 20 and 21 show the convergence in the relative error in the energy norm (57) for a single point along the curve $U_{h p}(t)$, at times $t=0.5 \mathrm{sec}$ and $t=1.0 \mathrm{sec}$, respectively. Again, we see that in the lower error ranges, the $h p$-GFEM delivers slightly better accuracy at a given element size. At time $t=0.5 \mathrm{sec}$ we see that both the $h p-G F E M$ and GFEM ${ }^{\mathrm{gl}}$ deliver the same convergence rates, $\beta$, and at time $t=1.0 \mathrm{sec}$ the $h p$ GFEM has a higher convergence rate, but the rate is still comparable with that obtained with the GFEM ${ }^{\mathrm{gl}}$. This behavior is, again, very similar to the steady-state case shown in Figure 15.

$$
\frac{\left\|u_{h p}(t)-u_{r e f}(t)\right\|_{E}}{\left\|u_{r e f}(t)\right\|_{E}}=\left(\frac{U_{h p}(t)-U_{r e f}(t)}{U_{r e f}(t)}\right)^{1 / 2}
$$

Analysis of Beam with Material Heterogeneity We now apply the transient GFEM $^{\mathrm{gl}}$ with Transient Formulation 2 to simulations involving material heterogeneity. Several different beam materials are investigated for the same beam model. In the first case, the beam is assumed to be made entirely of $A l$. In the second case, the beam is assumed to be made entirely of $\mathrm{SiC}$. In the third case, the beam is assumed to be a composite made up of $\mathrm{Al}$ and $\mathrm{SiC}$, with the volume fraction of $\mathrm{SiC}, V_{S i C}$, taken to be constant through the thickness of the beam, with $V_{S i C}=0.5$. Cases 4 and 5 assume a variation of $V_{S i C}$ through the thickness of the beam, according to the following power law

$$
V_{S i C}=V_{S i C}^{\text {bottom }}+\left(V_{S i C}^{\text {top }}-V_{S i C}^{\text {bottom }}\right)\left(\frac{y}{h}\right)^{q}
$$

where $V_{S i C}^{\text {bottom }}$ and $V_{S i C}^{\text {top }}$ are the volume fraction of silicon carbide at the top and bottom faces of the beam, respectively, taken as $V_{S i C}^{t o p}=1.0, V_{S i C}^{b o t t o m}=0.0 ; y$ is the $y$-coordinate of the material point, and $h$ is the height of the beam, $h=0.24 \mathrm{in}$. Simulations are run using $q=1,3$, corresponding to through-the-thickness variations of $V_{S i C}$ shown in Figure 22. A summary of the material composition of each case analyzed is provided in Table 5. 


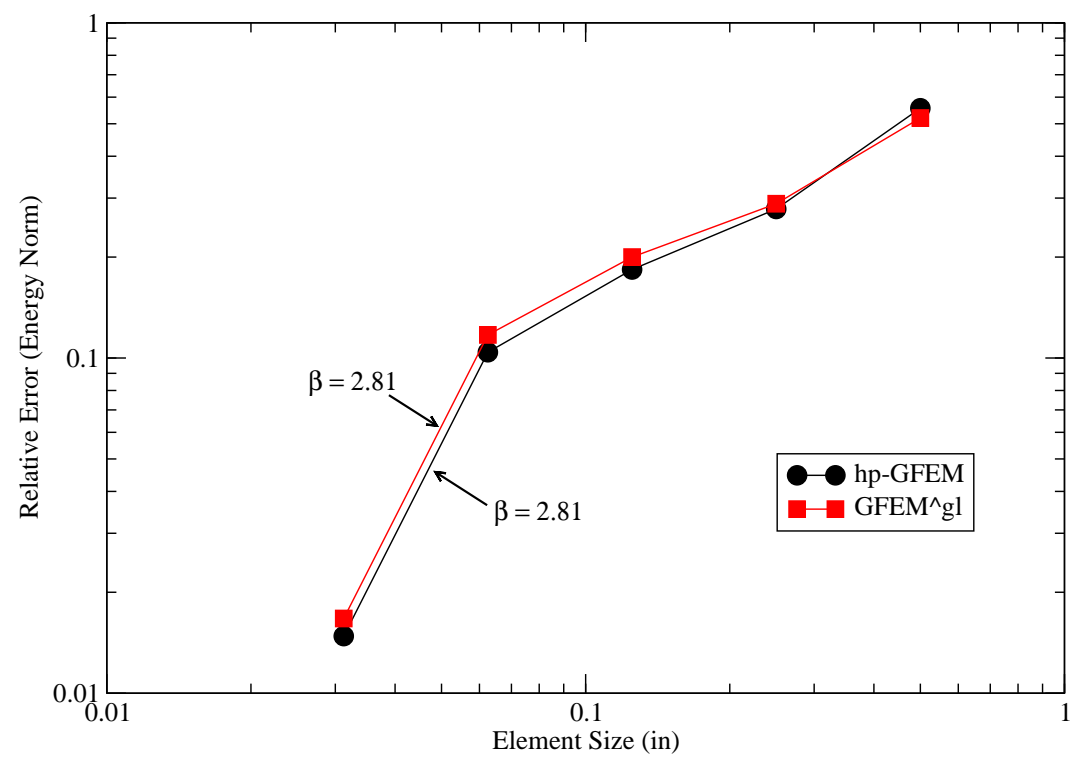

Figure 20: Convergence in energy norm for $h p$-GFEM and $\mathrm{GFEM}^{g l}$ solutions at $t=0.5 \mathrm{sec}$.

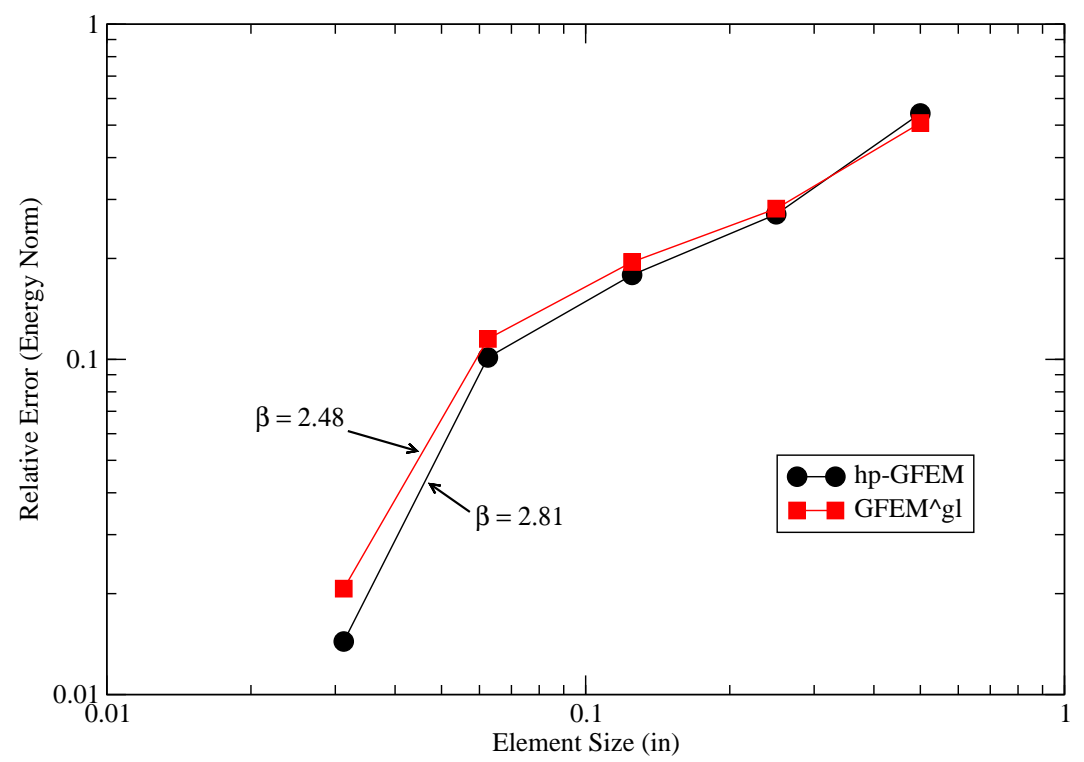

Figure 21: Convergence in energy norm for $h p$-GFEM and $\mathrm{GFEM}^{g l}$ solutions at $t=1.0$ sec.

Table 5: Material Composition for Each Case.

\begin{tabular}{ccc}
\hline Case & Material & $V_{S i C}$ \\
\hline 1 & $A l$ & 0.0 \\
2 & SiC & 1.0 \\
3 & $A l-S i C$ & 0.5 \\
4 & $A l-S i C$ & Power Law $(\mathrm{q}=1)$ \\
5 & $A l-S i C$ & Power Law $(\mathrm{q}=3)$ \\
\hline
\end{tabular}




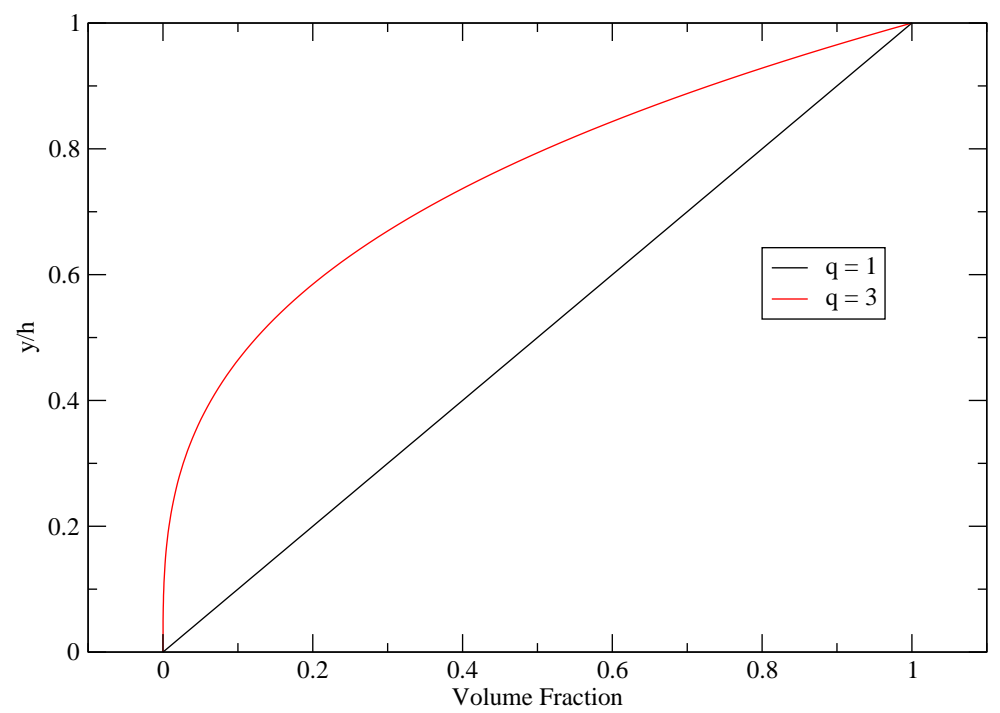

Figure 22: Illustrates the through-the-thickness variation of $V_{S i C}$ for the values of $q$ used in the simulations.

For the cases in which the beam is assumed to be a Functionally Graded Material (FGM) (cases 4 and 5), the values of the effective material properties, $(\rho c)_{e f f}$ and $\kappa_{\text {eff }}$ are homogenized using the rule-of-mixtures (59) and the Mori-Tanaka method (60), respectively [10, 24].

$$
\begin{gathered}
(\rho c)_{e f f}=V_{S i C} *(\rho c)_{S i C}+\left(1-V_{S i C}\right)(\rho c)_{A l} \\
\kappa_{e f f}=\kappa_{S i C}+\frac{\left(\kappa_{A l}-\kappa_{S i C}\right) *\left(1-V_{S i C}\right)}{1+V_{S i C} \frac{\kappa_{A l}-\kappa_{S i C}}{3 \kappa_{S i C}}}
\end{gathered}
$$

In all cases, reference solutions are computed using the same procedure described earlier for the $A l$ beam. Results obtained using the $G F E M^{\mathrm{gl}}$ for Cases 1 and 2 ( $\mathrm{Al}$ and $\mathrm{SiC}$ ), the two base materials selected for analysis are plotted in Figure 23. The figure shows the convergence in the $L_{2}^{\text {error }}$ norm of the internal energy for the $G F E M^{\mathrm{gl}}$. As can be seen from the figure, good convergence behavior is obtained for Cases 1 and 2.

Figure 24 shows the internal energy versus time curves for the first two cases. As we would expect, the material with a smaller thermal conductivity has steeper gradients in the solution, and thus more internal energy. In the figure, the GFEM $^{\mathrm{gl}}$ curves are not visible in the plot because they fall on top of $h p$-GFEM reference curves.

Results for the through-the-thickness temperature distributions are provided in Figure 25. The figure shows the convergence in the $L_{2}^{\text {error,temp }}(u(\boldsymbol{x}))$ value for the through-the-thickness temperature distributions at $t_{\text {final }}$ for Cases 1 and 2, as defined below

$$
L_{2}^{\text {error,temp }}(u(\boldsymbol{x}))=\frac{\left\|u\left(x, y, z^{n}\right)-u_{\mathrm{hp}}\left(x, y, z^{n}\right)\right\|_{2}}{\left\|u\left(x, y, z^{n}\right)\right\|_{2}}
$$

where the $n$ temperature values are taken at $(x, y, z)=\left(9.3,0.25, z^{n}\right)$, with $z^{n}=[0.0,0.2, \ldots, 0.24]$, and $\|\cdot\|_{2}$ is the discrete $L_{2}$ norm defined in (45). From the figure, it is apparent that good convergence behavior is obtained for this error parameter. Figure 26 shows the actual through-the-thickness temperature variations at $t_{\text {final }}$ for each of the 5 cases. For each of the plots provided, solid lines indicate reference curves generated with the $h p-G F E M$, and glyphs indicate $G F E M^{\mathrm{gl}}$ data. The $G F E M^{\mathrm{gl}}$ data fall on top of the reference $h p$ GFEM curves. 


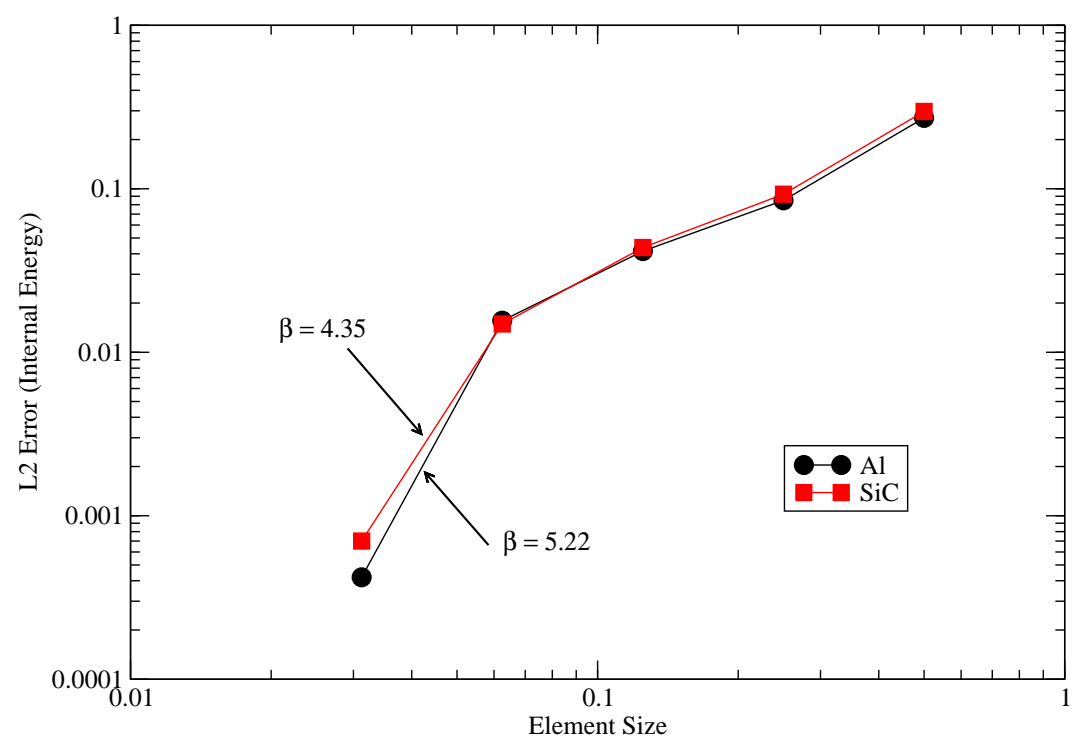

Figure 23: Convergence in $L_{2}^{\text {error }}(U(t))$ norm of the internal energy for the GFEM ${ }^{g l}$ as applied to Case 1 (Al) and Case 2 ( $\mathrm{SiC})$.

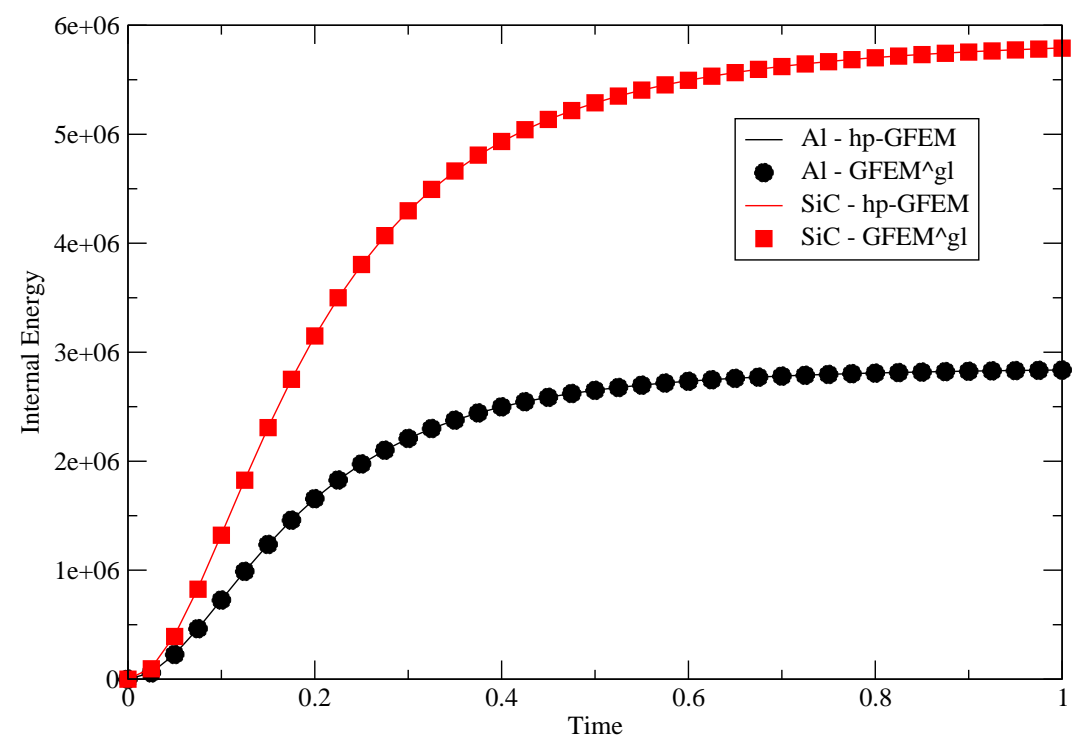

Figure 24: Internal energy versus time curves for Case 1 (Al) and Case 2 (SiC) computed with $\mathrm{GFEM}^{\text {gl }}$ and hpGFEM. Reference curves generated using hp-GFEM are shown using solid lines while the GFEM ${ }^{g l}$ data are shown using glyphs. 


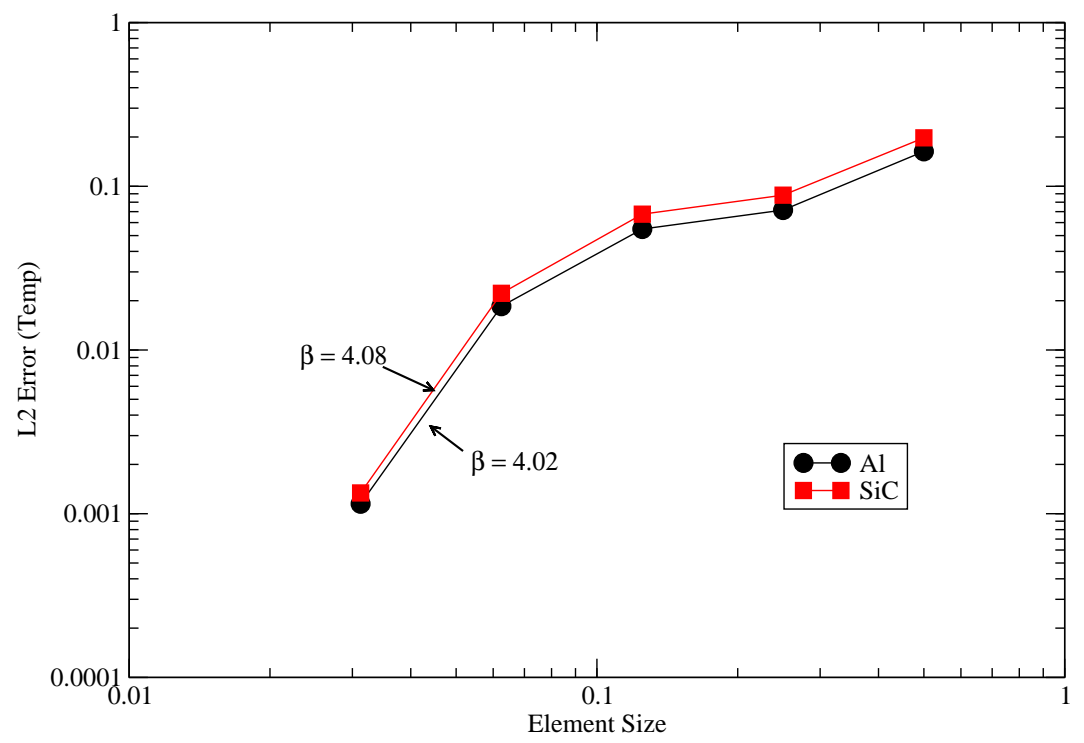

Figure 25: Convergence in the $L_{2}$ norm of through-the-thickness temperature distributions for the $\mathrm{GFEM}^{\text {gl }}$ as applied to Case 1 ( $\mathrm{Al})$ and Case $2(\mathrm{SiC})$.

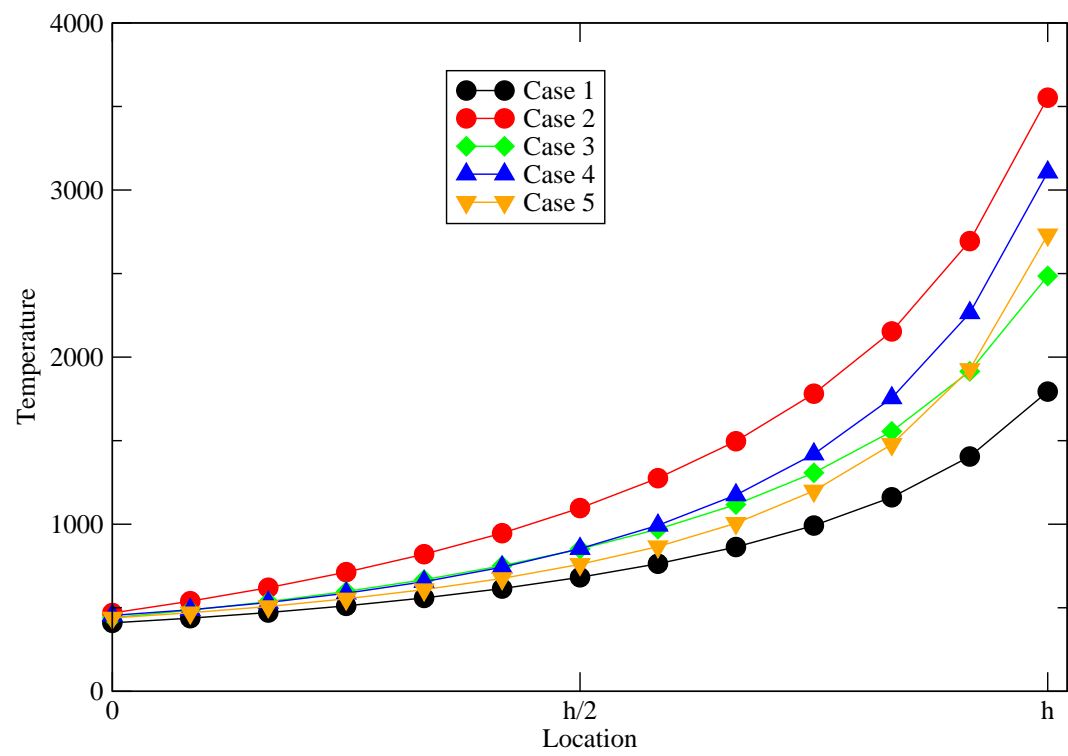

Figure 26: Through-the-thickness temperature distributions at $t_{\text {final }}$ for each of the 5 Cases. For each of the plots, solid lines indicate reference curves generated with the $h p$-GFEM, and glyphs indicate $\mathrm{GFEM}^{\text {gl }}$ data. 
Table 6 summarizes the $L_{2}^{\text {error }}$ norm of the internal energy, $U_{h p}(t)$, as well as the through-the-thickness temperature distributions at $t_{\text {final }}$ for each of the 5 Cases. As can be seen from the data, GFEM ${ }^{\text {gl }}$ results are in good agreement with the reference $h p$-GFEM results for each of the five cases in terms of internal energy versus time as well as for through-the-thickness temperature curves at $t_{\text {final }}$.

Table 6: $L_{2}^{\text {error }}$ values for each trial investigated. $L_{2}^{\text {error }}($ Temp $)$ is computed at $t_{\text {final }}$.

\begin{tabular}{cccc}
\hline Case & Material Type & $L_{2}^{\text {error }}($ Temp $)$ & $L_{2}^{\text {error }}($ Internal Energy)) \\
\hline 1 & $A l$ & $1.15 \mathrm{e}-3$ & $4.18 \mathrm{e}-4$ \\
2 & $S i C$ & $1.34 \mathrm{e}-3$ & $7.31 \mathrm{e}-4$ \\
3 & $V_{S i C}=0.5$ & $1.26 \mathrm{e}-3$ & $5.07 \mathrm{e}-4$ \\
4 & $q=1$ & $1.76 \mathrm{e}-3$ & $1.12 \mathrm{e}-2$ \\
5 & $q=3$ & $2.42 \mathrm{e}-3$ & $1.53 \mathrm{e}-2$ \\
\hline
\end{tabular}

\subsubsection{Effect of Time-Step Size}

In this section we analyze the beam with material properties corresponding to $A l$, or Case 1 , and varying time step sizes, $\Delta t$. For the $G F E M^{\mathrm{gl}}$ simulations, there is a lag in the boundary conditions applied to the local domain due to the fact that in the generation of the enrichment function used at the global domain at time $t^{n+1}$, the Dirichlet BCs are taken from the global solution from time $t^{n}$, whereas the Neumann BCs and heat source are taken from the prescribed data at time $t^{n+1}$. As such it is reasonable to investigate the accuracy of the $G F E M^{\mathrm{gl}}$ results relative to those generated with $h p$-GFEM for different time-step sizes, so as to investigate whether or not the lag in the boundary conditions resulting from the size of $\Delta t$ has a negative impact of the $G F E M^{\mathrm{gl}}$ solution quality. Figure 27 shows the value of $L_{2}^{\text {Diff }}$ for the $G F E M^{\mathrm{gl}}$ and $h p-G F E M$ simulations. In this instance we are not plotting temporal convergence with respect to the size of $\Delta t$, as for each point on the plot, the time-step size is the same for the $G F E M^{\mathrm{gl}}$ simulation considered, as well as for the $h p-G F E M$ simulation used as reference.

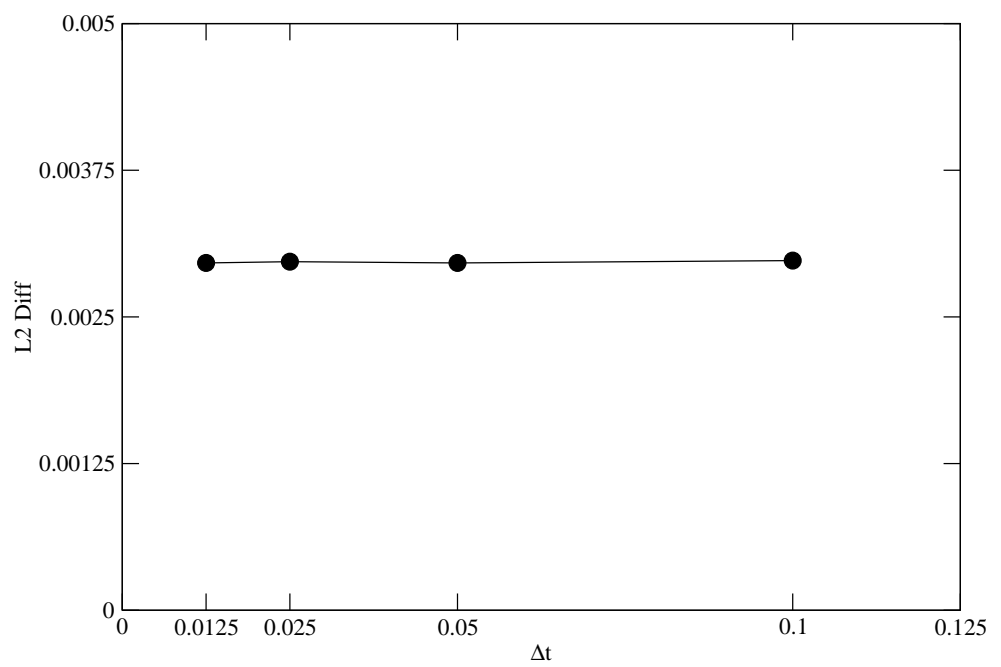

Figure 27: Plot of $L_{2}^{D i f f}$ for $h p$-GFEM and $\mathrm{GFEM}^{g l}$ simulations with different size $\Delta t$. 
In this instance, we define the value of $L_{2}^{\text {Diff }}$ as

$$
L_{2}^{\text {Diff }}=\frac{\left\|U_{h p}(t)-U_{g l}(t)\right\|_{2}}{\left\|U_{h p}(t)\right\|_{2}}
$$

where $U_{h p}(t)$ and $U_{g l}(t)$ are the internal energy computed with the $h p$-GFEM and GFEM ${ }^{\mathrm{gl}}$, respectively, and $\|\cdot\|_{2}$ is the discrete $L_{2}$ norm defined in (45). Both the $h p-G F E M$ and GFEM ${ }^{\mathrm{gl}}$ curves are generated on meshes with 7 levels of refinement around the laser flux location. From the plot it can be seen that the $L_{2}^{\text {Diff }}$ value is, at least for this problem, relatively insensitive to the time step size used, and the lag in the boundary conditions applied to the local problem from the global solution at $t^{n}$ do not cause a problem in terms of the quality of the local solution, even when a larger $\Delta t$ is used.

It is reasonable to assume that there is a $\Delta t$ value which is large enough to deteriorate the local boundary condition quality and to significantly impact the accuracy of the $G F E M^{\mathrm{gl}}$ solution. It is not obvious however, how this $\Delta t$ value governing the severity of the boundary condition lag compares with the value of $\Delta t$ required for sufficient accuracy of the transient simulation, regardless of whether the $G F E M^{\mathrm{gl}}$ or the $h p$ GFEM is used. This type of convergence study is beyond the scope of the current work, but will likely be the focus of future investigation.

\subsubsection{Effect of Time-Rise Constant, $\gamma$}

We now seek to investigate the effect of the time-rise constant, $\gamma$ on the ability of the transient $G F E M^{\mathrm{gl}}$ to deliver accurate results. The material properties used in this section correspond to $A l$, referred to previously as Case 1. Figure 28 shows the spatial and temporal variations for the Gaussian flux when $\gamma=50$.

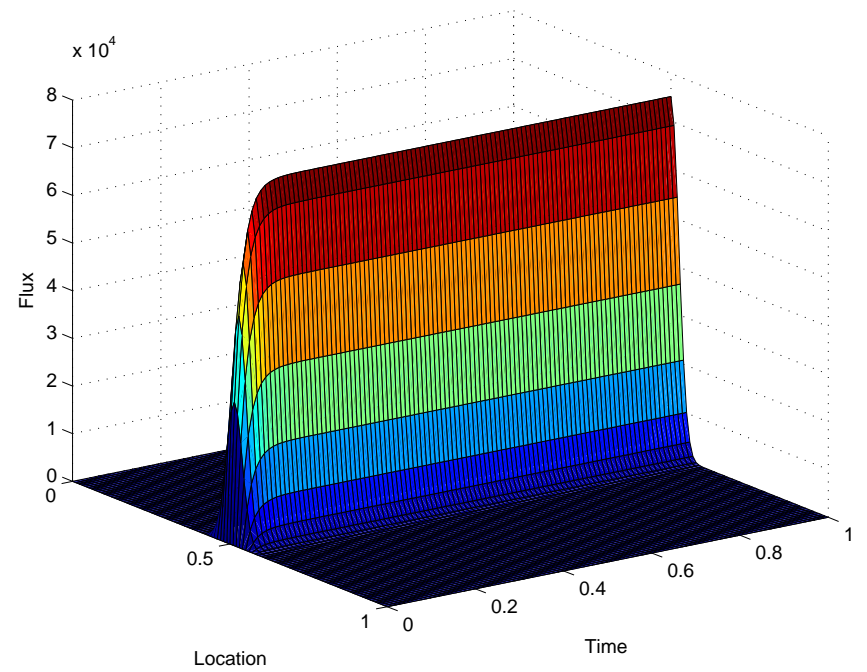

Figure 28: Spatial and temporal variation of the Gaussian laser flux, $(\gamma=50)$.

In this instance, the intensity of the flux ramps up much more quickly in time as compared to Figure 12, resulting in a larger gradient in the internal energy versus time reference curve for the model problem, as shown in Figure 29. In the figure, a reference curve generated using $h p-G F E M$ is shown using a solid line while the $G F E M^{\mathrm{gl}}$ data are shown using glyphs. The FFEM $^{\mathrm{gl}}$ data for $\gamma=50$ once again falls on top of the reference curve. The curves corresponding to $\gamma=10$ are also provided to illustrate the difference in the 
evolution of the internal energy with respect to an increase in $\gamma$. In both instances, $\gamma=10$ and $\gamma=50,9$ levels of localized refinement are used.

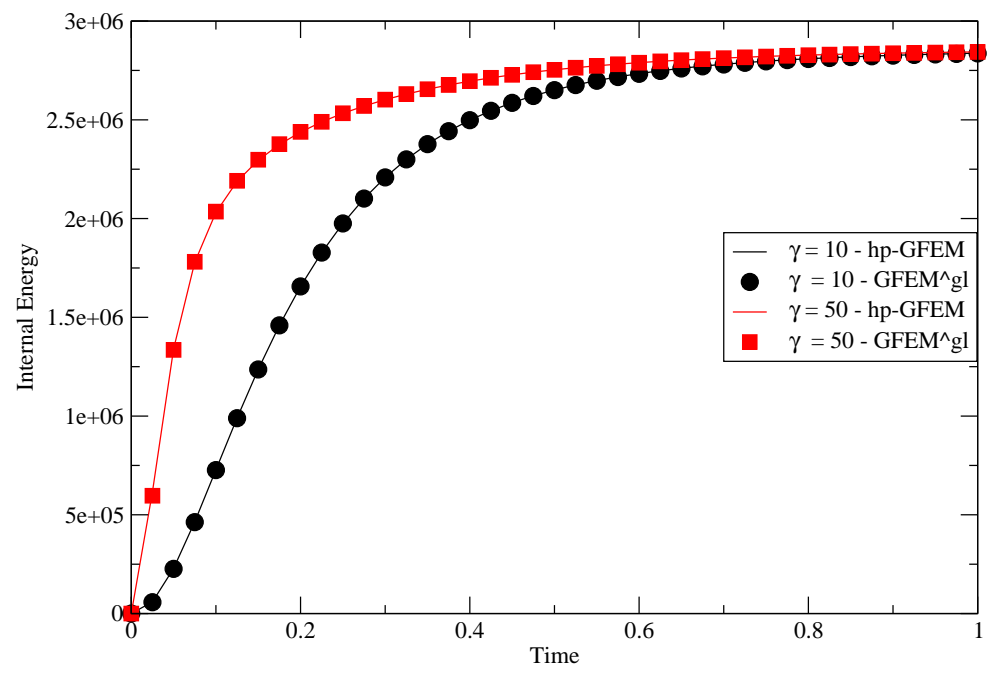

Figure 29: Internal energy vs time curves for solutions obtained with time-rise constant $\gamma=10$ and $\gamma=50$. Reference curves generated using hp-GFEM are shown using solid lines while the $\mathrm{GFEM}^{g l}$ data are shown using glyphs.

The convergence in the $L_{2}^{\text {error }}$ norm of the internal energy is shown in Figure 30 for $\gamma=10$ and $\gamma=50$. From the plots it can be seen that in both cases good convergence behavior is obtained, with differences only at very low error values. In both cases the $L_{2}^{\text {error }}$ value is driven below one percent relative difference.

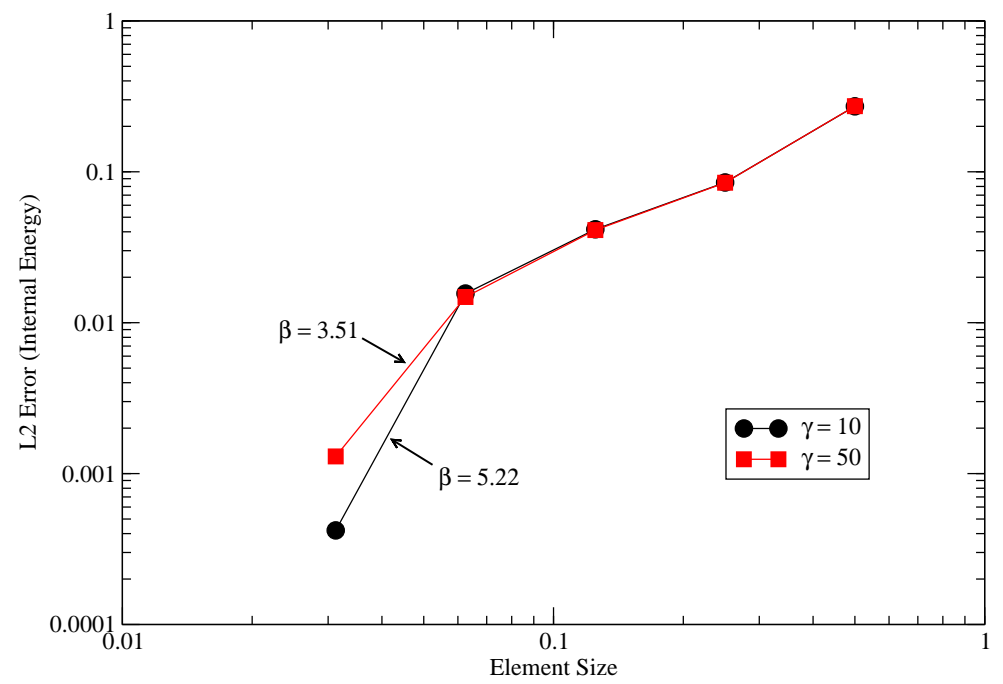

Figure 30: Convergence in the $L_{2}^{\text {error }}$ norm of the internal energy for $G F E M^{g l}$ as a function of element size in local problem.

The convergence in the $L_{2}^{\text {error }}$ norm of the through-the-thickness temperature distribution is shown in Figure 31 for $\gamma=10$ and $\gamma=50$. The temperatures are taken from the same location as previously noted. From the plots it is again seen that good convergence behavior is obtained, with differences only at very low error values. In both cases the $L_{2}^{\text {error }}$ value is once again driven below one percent.

The evolution of the through-the-thickness temperature distributions in time are shown in Figures 32 and 33 for $\gamma=10,50$, respectively. From the figures it can be seen that for $\gamma=50$ the temperature distributions 


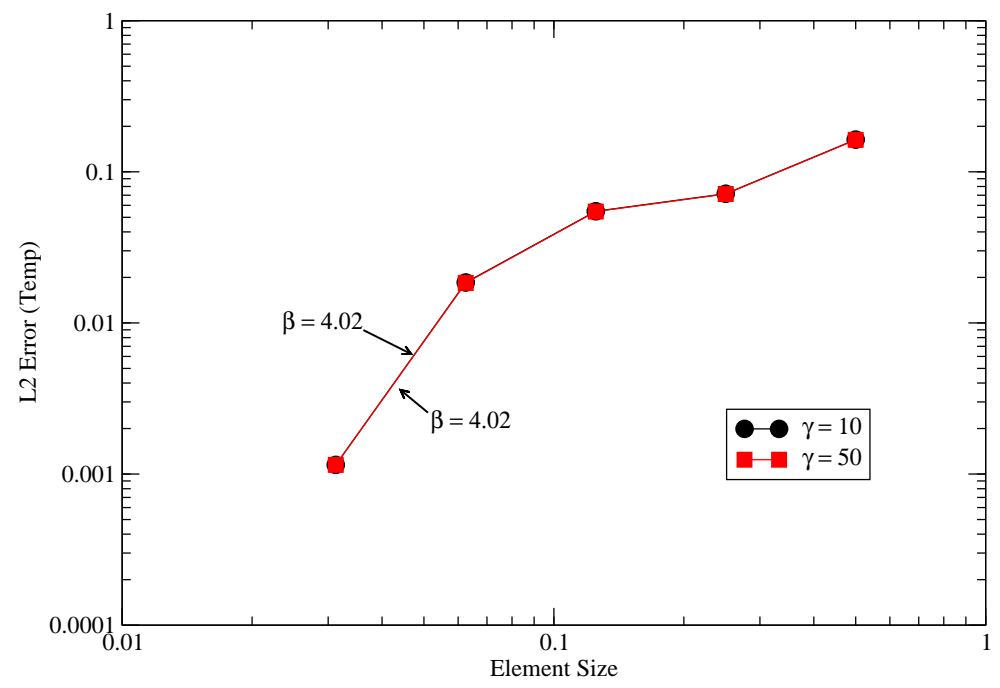

Figure 31: Convergence in the $L_{2}^{\text {error }}$ norm of through-the-thickness temperature distribution at $t_{\text {final }}$ as a function of element size in local problem.

at $t=0.3,0.65$ seconds are closer to the steady-state curves than those obtained with $\gamma=10$, as would be expected.

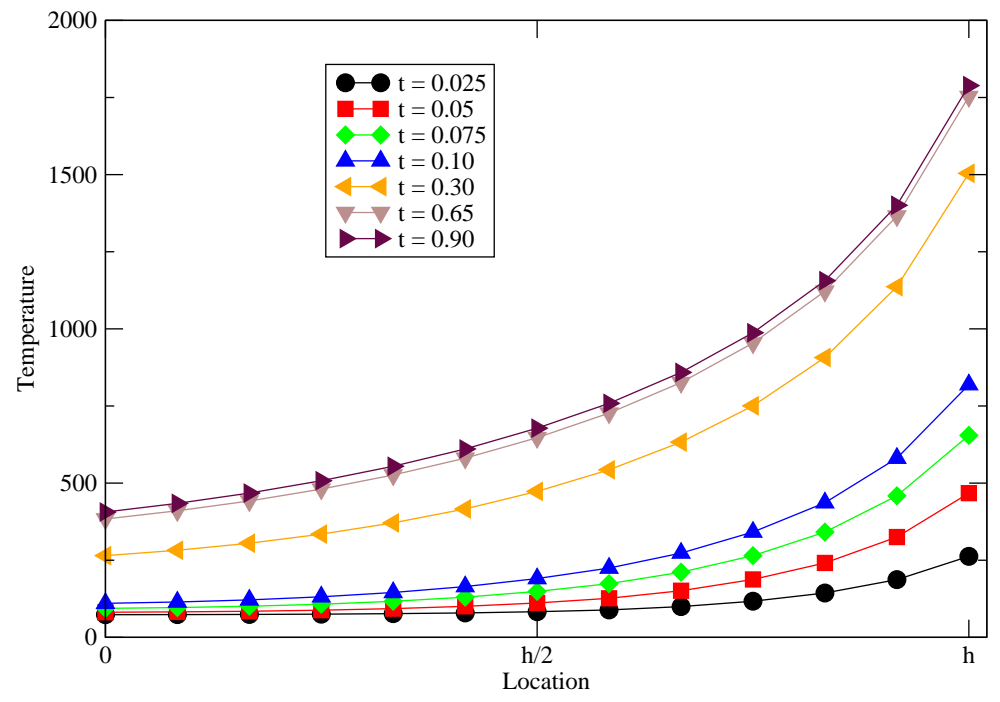

Figure 32: Evolution of the through-the-thickness temperature distribution for the beam with $\gamma=10$. 


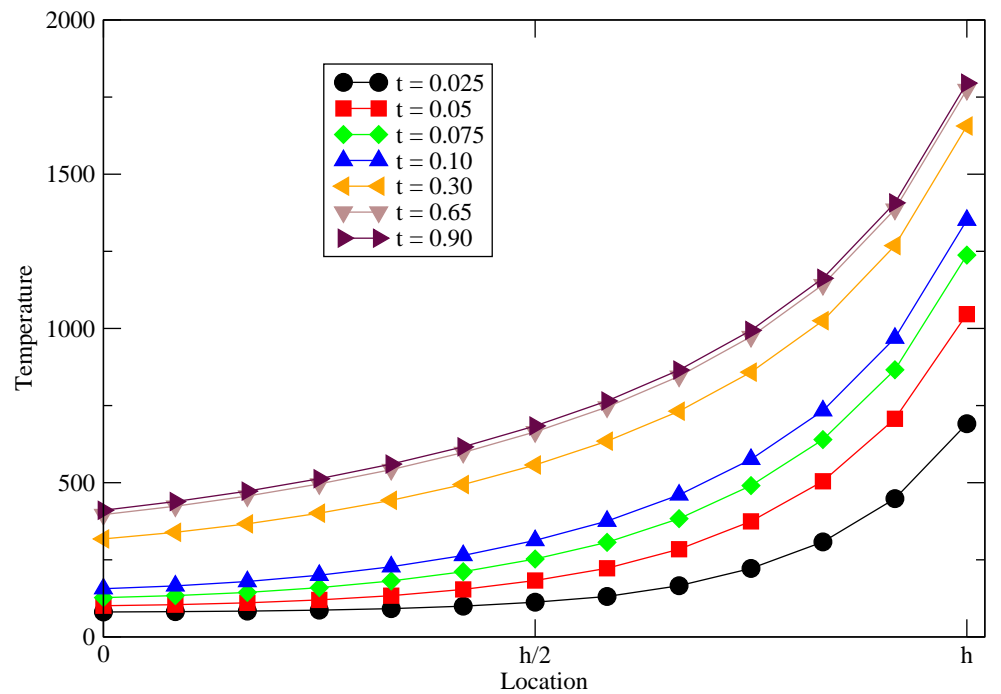

Figure 33: Evolution of the through-the-thickness temperature distribution for the beam with $\gamma=50$. 


\subsection{Numerical Experiment 3: Beam Subjected to Moving Laser Flux}

In this section we analyze the same beam model as in the previous, with material properties corresponding to $A l$, or Case 1 in the previous section. In this example, the applied laser flux increases in time, as well as moves in space, as shown in Figure 34.

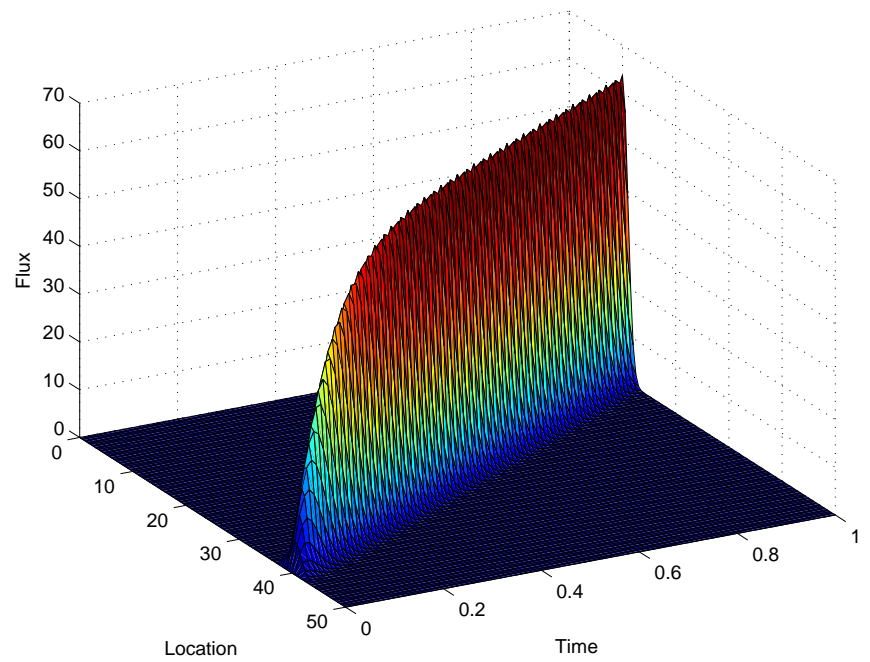

Figure 34: Flux applied to the beam.

The applied Gaussian laser flux function now takes the form:

$$
\begin{gathered}
\bar{f}(\boldsymbol{x}, t)=I_{0} * f(t) * \frac{1}{2 \pi a^{2}} * G(\boldsymbol{x}, b(t), a) \\
f(t)=1-\exp (-\gamma * t) \\
G(\boldsymbol{x}, b(t), a)=\exp \left(\frac{-(x-b(t))^{2}}{2 a^{2}}\right) \\
b(t)=b_{0}+V t
\end{gathered}
$$

In the above equations, the constants take the values: $I_{0}=295 \frac{f t-l b f}{s}, a=0.025 \mathrm{in}, \gamma=10.0 \mathrm{~s}^{-1}, b_{0}=9.25 \mathrm{in}$ and $V=0.5 \frac{\mathrm{in}}{\mathrm{sec}}$. The reference solution in this section is generated using $h p-G F E M$ with high levels of local refinement and cubic shape functions, resulting in a model with 433,635 dofs. The GFEM $^{\text {gl }}$ solutions are once again generated using meshes with only 1,020 dofs and solution accuracy is again improved through the use of $h$-extensions in the local problem.

Figure 35 shows the internal energy versus time curves for the FFEM $^{\mathrm{gl}}$ simulation as well as the $h p-G F E M$ reference curve. The $G F E M^{\mathrm{gl}}$ curve is again difficult to see because it falls on top of the reference curve. Nine levels of mesh refinement were applied to the local problem used with the $G F E M^{\mathrm{gl}}$ The $L_{2}^{\text {error }}$ error norm of the internal energy for the $G F E M^{\mathrm{gl}}$ simulation is $6.01 e-4$, indicating excellent agreement between the $G F E M^{\mathrm{gl}}$ solution and the reference $h p-G F E M$ solution.

Figures 36 and 37 show snap-shots of the solution at different times throughout the simulation for $G F E M^{\text {gl }}$ 


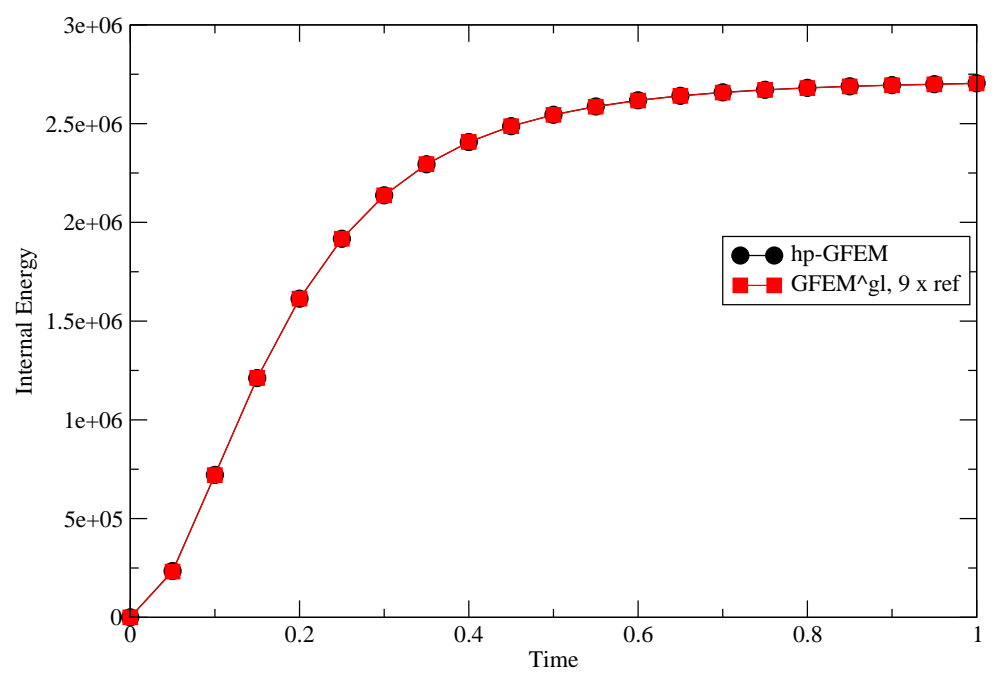

Figure 35: Internal energy vs time curves for solutions obtained with a moving source.

solutions and $h p$-GFEM solutions, respectively. It is of greatest interest to highlight the ability of the GFEM $^{\mathrm{gl}}$ to resolve the transient, moving, thermal spike on elements which are significantly larger than the width of the spike itself, as shown in Figure 36. By contrast, Figure 37 illustrates the ability of the $h p$-GFEM to resolve the moving spike as well, but with the requirement of a significant increase in the mesh density. As such, there is great potential for a significant increase in the computational efficiency for transient simulations using the FFEM $^{\mathrm{gl}}$.

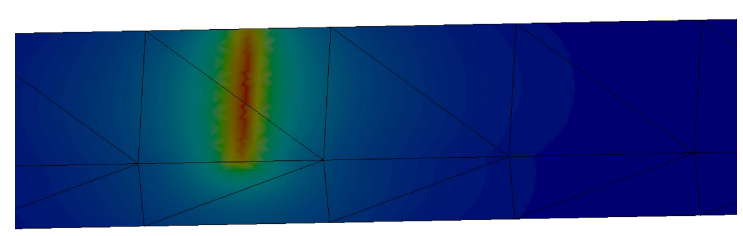

(a) $\mathrm{t}=0.05$

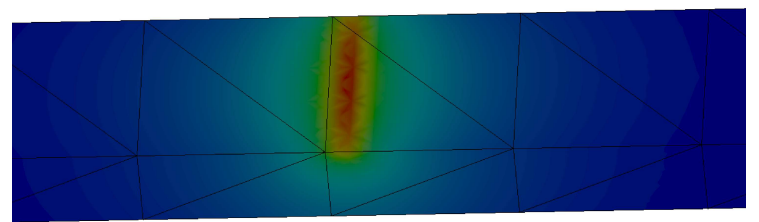

(c) $\mathrm{t}=0.65$

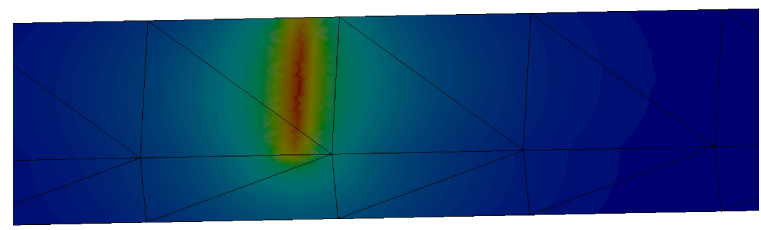

(b) $t=0.35$

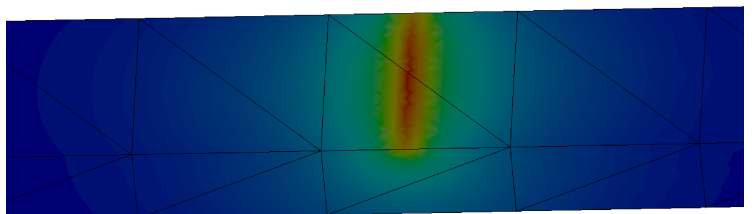

(d) $\mathrm{t}=0.95$

Figure 36: Snap-shots in time for the transient $\mathrm{GFEM}^{\text {gl }}$ simulation for the beam with a moving laser flux. 


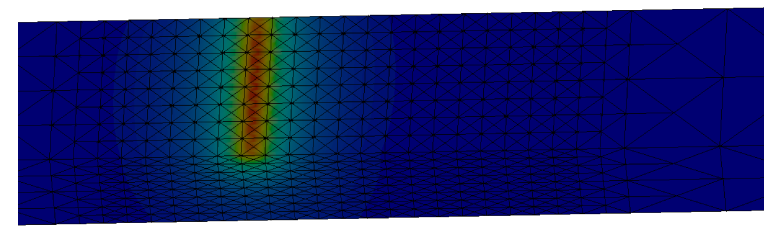

(a) $\mathrm{t}=0.05$

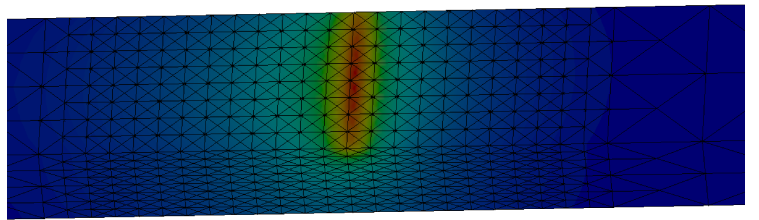

(c) $\mathrm{t}=0.65$

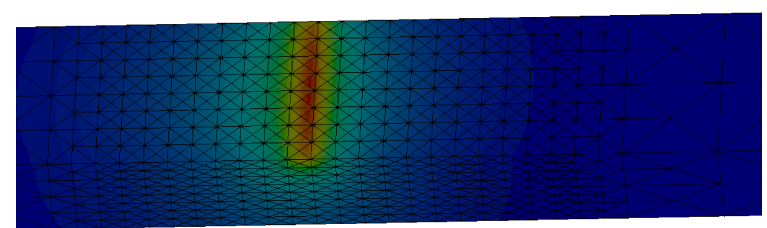

(b) $t=0.35$

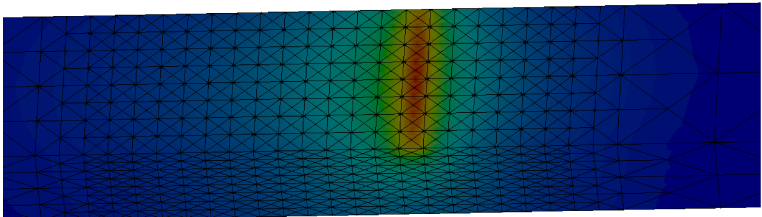

(d) $\mathrm{t}=0.95$

Figure 37: Snap-shots in time for the transient hp-GFEM simulation for the beam with a moving laser flux. 


\section{Conclusions}

In this paper, the generalized FEM with global-local enrichments $\left(\right.$ GFEM $\left.^{\mathrm{gl}}\right)[14,17,25,32]$ is formulated for transient heat transfer problems with solutions exhibiting highly-localized, sharp thermal gradients. The proposed method enables the analysis of this class of problems using uniform, coarse, global meshes. This has several computational implications as discussed in Section 7.

(i) the possibility of capturing localized, transient solution features using uniform, coarse, global meshes. This removes, for example, the need to refine global meshes that are usually complex and very large, the re-meshing of which is non-trivial between subsequent time-steps;

(ii) no transient effects need to be considered in the local domains;

(iii) with the proper discretization order (temporal, spatial) the $G F E M^{\mathrm{gl}}$ produces results which are in very good agreement with the reference curves generated using $h p$-GFEM and significantly more degrees of freedom;

(iv) the FFEM $^{\mathrm{gl}}$ delivers accurate results in terms of the evolution of the internal energy as a function of time, as well as in the resulting through-the-thickness temperature distributions. The latter of which is important for design considerations, particularly if thermo-mechanical coupling is considered. Thermo-mechanical coupling, while not addressed in this work, is a topic to be investigated in future work;

(v) the size of the enriched global problem does not depend on the size or discretization used in the local problems;

(vi) the accuracy of the $G F E M^{\mathrm{gl}}$ is relatively insensitive to the time-rise constant of the applied surface flux;

(vii) the $G F E M^{\mathrm{gl}}$ uses a large amount of information which can be calculated once and re-used at each subsequent time-step, yielding the potential for significant improvement in the computational efficiency. This potential increase in efficiency is the current focus upon the completion of this work;

(viii) the accuracy of the $G F E M^{\mathrm{gl}}$ is relatively insensitive to the size of $\Delta t$, and the resulting lag in Dirichlet boundary conditions applied in the local problems;

Acknowledgments: The authors gratefully acknowledge the contributions of the Midwest Structural Sciences Center (MSSC) at the University of Illinois at Urbana-Champaign. The Center is supported by the U.S. Air Force Research Laboratory Air Vehicles Directorate under contract number FA8650-06-2-3620. The authors also wish to thank Prof. Uday Banerjee from Syracuse University for fruitful discussions during the course of this research.

\section{References}

[1] D. N. Arnold, A. Mukherjee, and L. Pouly. Locally adapted tetrahedral meshes using bisection. SIAM Journal of Scientific Computing, 22(2):431-448, 2000.

[2] I. Babuska. The finite element method with penalty. Mathematics of Computation, 27(122):221-228, 1973. 
[3] I. Babuška and J.M. Melenk. The partition of unity finite element method. International Journal for Numerical Methods in Engineering, 40:727-758, 1997.

[4] I. Babuška, G. Caloz, and J.E. Osborn. Special finite element methods for a class of second order elliptic problems with rough coefficients. SIAM Journal on Numerical Analysis, 31(4):945-981, 1994.

[5] I. Babuška, F. Ihlenburg, E. Paik, and S. Sauter. A generalized finite element method for solving the Helmholtz equation in two dimensions with minimal pollution. Computer Methods in Applied Mechanics and Engineering, 128(3-4):325-360, 1995.

[6] I. Babuška, U. Banerjee, and J.E. Osborn. Generalized finite element methods - Main ideas, results and perspective. International Journal of Computational Methods, 1(1):67-103, 2004.

[7] E. Bansch. Local mesh refinement in 2 and 3 dimensions. Impact of Computing in Science and Engineering, 3:181-191, 1991.

[8] J. Chessa and T. Belytschko. An enriched finite element method and level sets for axisymmetric twophase flow with surface tenstion. International Journal for Numerical Methods in Engineering, 58: 2041-2064, 2003.

[9] J. Chessa and T. Belytschko. Arbitrary discontinuities in space-time finite elements by level-stes and x-fem. International Journal for Numerical Methods in Engineering, 61:2595-2614, 2004.

[10] H. K. Ching and J. K. Chen. Thermomechanical analysis of functionally graded composites under laser heating by the MLPG method. Computer Modeling in Engineering and Sciences, 13(3):199$217,2006$.

[11] D. D'Ambrosio. Numerical prediction of laminar shock/shock interactions in hypersonic flow. Journal of Spacecraft and Rockets, 40(2):153-161, 2003.

[12] C.A. Duarte. The hp Cloud Method. PhD dissertation, The University of Texas at Austin, December 1996. Austin, TX, USA.

[13] C.A. Duarte and I. Babuška. Mesh-independent directional p-enrichment using the generalized finite element method. International Journal for Numerical Methods in Engineering, 55(12):1477-1492, 2002. http://dx.doi.org/10.1002/nme.557.

[14] C.A. Duarte and D.-J. Kim. Analysis and applications of a generalized finite element method with global-local enrichment functions. Computer Methods in Applied Mechanics and Engineering, 197 (6-8):487-504, 2008. http://dx.doi.org/10.1016/j.cma.2007.08.017.

[15] C.A. Duarte, I. Babuška, and J.T. Oden. Generalized finite element methods for three dimensional structural mechanics problems. Computers and Structures, 77:215-232, 2000.

[16] C.A. Duarte, O.N. Hamzeh, T.J. Liszka, and W.W. Tworzydlo. A generalized finite element method for the simulation of three-dimensional dynamic crack propagation. Computer Methods in Applied Mechanics and Engineering, 190(15-17):2227-2262, 2001. http://dx.doi.org/10.1016/S00457825(00)00233-4.

[17] C.A. Duarte, D.-J. Kim, and I. Babuška. Chapter: A global-local approach for the construction of enrichment functions for the generalized fem and its application to three-dimensional cracks. In V.M.A. Leitão, C.J.S. Alves, and C.A. Duarte, editors, Advances in Meshfree Techniques, volume 5 of Computational Methods in Applied Sciences, The Netherlands, 2007. Springer. ISBN 978-1-4020-6094-6. 
[18] C.A.M. Duarte and J.T. Oden. Hp clouds-A meshless method to solve boundary-value problems. Technical Report 95-05, TICAM, The University of Texas at Austin, May 1995.

[19] C.A.M. Duarte and J.T. Oden. Hp clouds - An $h p$ meshless method. Numerical Methods for Partial Differential Equations, 12:673-705, 1996.

[20] C.A.M. Duarte and J.T. Oden. An $h p$ adaptive method using clouds. Computer Methods in Applied Mechanics and Engineering, 139:237-262, 1996.

[21] P. P. Friedmann, K. G. Powell, J. J Mcnamara, B. J. Thuruthimattam, and R. Bartels. Hypersonic aerothermoelastic studies for reusable launch vehicles, 2004. AIAA 2004-1590.

[22] P. P. Friedmann, K. G. Powell, J. J Mcnamara, B. J. Thuruthimattam, and R. Bartels. Three-dimensional aeroelastic and aerothermoelastic behavior in hypersonic flow, 2005. AIAA 2005-2175.

[23] T. P. Fries and A. Zilian. On time integration in the xfem, 2009.

[24] H. Hatta and M. Taya. Effective thermal conductivity of a misoriented short fiber composite. Journal of Applied Physics, 58(7):2478, 1985.

[25] D.-J. Kim, C.A. Duarte, and J.P. Pereira. Analysis of interacting cracks using the generalized finite element method with global-local enrichment functions. Journal of Applied Mechanics, 75(5):051107, 2008. doi: 10.1115/1.2936240. URL http://link.aip.org/link/?AMJ/75/051107/1.

[26] L. E. Lindgren. Numerical modeling of welding. Computer Methods in Applied Mechanics and Engineering, 195:6710-6736, 2006.

[27] J.M. Melenk. On Generalized Finite Element Methods. PhD thesis, The University of Maryland, 1995.

[28] J.M. Melenk and I. Babuška. The partition of unity finite element method: Basic theory and applications. Computer Methods in Applied Mechanics and Engineering, 139:289-314, 1996.

[29] R. Merle and J. Dolbow. Solving thermal and phase change problem with the eXtended finite element method. Computational Mechanics, 28:339-350, 2002.

[30] J. R. Moselle, A. R. Wieting, M.S. Holden, and C. Glass. Studies of aerothermal loads generated in regions of shock/shock interaction in hypersonic flow, 1988. AIAA-88-0477.

[31] J.T. Oden, C.A. Duarte, and O.C. Zienkiewicz. A new cloud-based $h p$ finite element method. Computer Methods in Applied Mechanics and Engineering, 153:117-126, 1998.

[32] P. O'Hara, C.A. Duarte, and T. Eason. Generalized finite element analysis of three-dimensional heat transfer problems exhibiting sharp thermal gradients. Computer Methods in Applied Mechanics and Engineering, 198(21-26):1857-1871, 2009. http://dx.doi.org/10.1016/j.cma.2008.12.024.

[33] Patrick James O'Hara. A Multi-Scale Generalized Finite Element Method for Sharp, Transient Thermal Gradients. PhD thesis, University of Illinois at Urbana-Champaign, 2010.

[34] M. E. Plesha, R. D. Cook, D. S. Malkus, and R. J. Witt. Concepts and Applications of Finite Element Analysis. John Wiley \& Sons, New York, 2002.

[35] J. N. Reddy and D. K. Gartling. The Finite Element Method in Heat Transfer and Fluid Dynamics. CRC Press LLC, Boca Raton, Florida, 2001. 
[36] A. Simone, C.A. Duarte, and E. van der Giessen. A generalized finite element method for polycrystals with discontinuous grain boundaries. International Journal for Numerical Methods in Engineering, 67 (8):1122-1145, 2006. http://dx.doi.org/10.1002/nme.1658.

[37] T. Strouboulis, K. Copps, and I. Babuška. The generalized finite element method: An example of its implementation and illustration of its performance. International Journal for Numerical Methods in Engineering, 47(8):1401-1417, 2000.

[38] T. Strouboulis, K. Copps, and I. Babuška. The generalized finite element method. Computer Methods in Applied Mechanics and Engineering, 190:4081-4193, 2001.

[39] E. A. Thornton, A. R. Wieting, and K. Morgan. Application of integrated fluid-thermal-structural analysis methods. Journal of Thin-Walled Structures, 11:1-23, 1991.

[40] T. L. Turner and R. L. Ash. Analysis of the thermal environment and thermal response associated with thermal-acoustic testing, 1990. AIAA-90-0975-CP.

[41] F. P. van der Meer, R. Al-Khoury, and L. J. Sluys. Time-dependent shape functins for modeling highly transient geothermal systems. International Journal for Numerical Methods in Engineering, 77:240260, 2009.

[42] H. Waisman and T. Belytschko. Parametric enrichment adaptivity by the extended finite element method. International Journal for Numerical Methods in Engineering, 73:1671-1692, 2008.

[43] A. R. Wieting. Experimental study of shock wave interference heating on a cylindrical leading edge, 1987. NASA Technical Memorandum 100484.

[44] A. H. Yaghi, T. H. Hyde, A. A. Becker, and W. Sun. Finite element simulation of welding and residual stresses in a p91 steel pipe incorporating solid-state phase transformation and post-weld heat treatment. Journal of Strain Analysis for Engineering Design, 43:275-293, 2008.

[45] G. Yu, K. Masubichi, T. Maekawa, and N. M. Patrikalakis. A finite element model for metal forming by laser line heating. Massachusetts Institue of Technology, Cambridge, MA.

[46] G. Yu, K. Masubichi, T. Maekawa, and N. M. Patrikalakis. Fem simulation of laser forming of metal plates. Journal of Manufacturing Science and Engineering, 123:405-410, 2001.

[47] O. C. Zienkiewicz, R. L. Taylor, and J. Z. Zhu. The Finite Element Method: Its Basis and Fundamentals. Elsevier Butterworth-Heinemann, 2005. 